\title{
FINITE JET DETERMINATION OF CONSTANTLY DEGENERATE CR EMBEDDINGS
}

\author{
PETER EBENFELT AND BERNHARD LAMEL
}

\begin{abstract}
We prove finite jet determination results for smooth CR embeddings which are of constant degeneracy, using the method of complete systems. As an application, we derive a reflection principle for mappings between a Levi-nondegenerate hypersurface in $\mathbb{C}^{N}$ and a Levi-nondegenerate hypersurface in $\mathbb{C}^{N+1}$. We also give an independent proof of the reflection principle for mappings between strictly pseudoconvex hypersurfaces in any codimension due to Forstneric [6].
\end{abstract}

\section{INTRODUCTION}

In this paper, we shall derive a complete system of differential equations for CR mappings between a smooth hypersurface in $\mathbb{C}^{N}$ and a smooth hypersurface in $\mathbb{C}^{\hat{N}}$ (see e.g. Theorem $\mathbb{8}$ ). This system of differential equations will then be used to prove finite determination results (Theorems 1, 2, and 7) for such mappings. For historical information and background on the finite determination problem, we refer the reader to the introduction of [5] or the survey article [1. As an application of our main results, we also prove a new reflection principle for smooth mappings between real-analytic Levi nondegenerate hypersurfaces (Corollary 5).

To formulate our results precisely, we shall need to introduce some notation. Let $M$ be a real hypersurface in $\mathbb{C}^{N}, N \geq 2$, and let us denote by $\mathcal{V} \subset \mathbb{C} T M$ its $\mathrm{CR}$ bundle; the reader is referred e.g. to 22 for basic notions and facts about CR manifolds. Recall that a mapping $f: M \rightarrow \mathbb{C}^{k}$ is called CR if $f_{*}(\mathcal{V}) \subset T^{0,1} \mathbb{C}^{k}$, where $f_{*}$ denotes the induced mapping of the tangent spaces (the push forward). This is equivalent to saying that, near every point $p \in M$, there is a neighborhood $U \subset M$ of $p$ in which

$$
L_{\bar{a}} f_{j}=0, \quad a=1, \ldots, n, j=1, \ldots k,
$$

where $n=N-1$ and $L_{\overline{1}}, \ldots, L_{\bar{n}}$ is a basis for the CR vector fields in $U$. We use here the convention that e.g. $L_{\bar{a}}=\overline{L_{a}}$ so that the $L_{1}, \ldots, L_{n}$ form a basis for the vector fields valued in $\overline{\mathcal{V}}$ near $p$ on $M$.

Suppose that $\hat{M} \subset \mathbb{C}^{\hat{N}}$ is a real hypersurface and $f: M \rightarrow \mathbb{C}^{\hat{N}}$ is a CR mapping sending $M$ into $\hat{M}$. Let $p$ be a point in $M$ and $\hat{\rho}$ a local defining function for $\hat{M}$ near $\hat{p}:=f(p) \in \hat{M}$. The mapping $f$ is called transversal at $p$ if $f_{*}\left(\mathbb{C} T_{p} M\right)$ is not contained in the subspace (of codimension 1) $\hat{\mathcal{V}}_{\hat{p}}+\overline{\hat{\mathcal{V}}}_{\hat{p}} \subset \mathbb{C} T_{\hat{p}} \hat{M}$. One can show (see section 3.4) that if $f$ is transversal and $M$ is Levi nondegenerate at $p \in M$, then $f$ is in fact a local embedding near $p$.

Following Lamel [9], we define an increasing sequence of subspaces $E_{k}(q) \subset \mathbb{C}^{\hat{N}}$, for $q \in M$ near $p$, associated to the germ of the mapping $f:(M, q) \rightarrow \hat{M}$ as follows. Let $L_{\overline{1}}, \ldots, L_{\bar{n}}$ be a basis for the CR vector fields on $M$ near $p$ as above and define

$$
E_{k}(q):=\operatorname{span}\left\{L^{\bar{J}}\left(\hat{\rho}_{Z^{\prime}} \circ f\right)(q): J \in\left(\mathbb{Z}_{+}\right)^{n},|J| \leq k\right\} \subset \mathbb{C}^{\hat{N}},
$$

1991 Mathematics Subject Classification. 32H02.

The first author is a Royal Swedish Academy of Sciences Research Fellow supported by a grant from the Knut and Alice Wallenberg Foundation and also by DMS-0100110. The second author was supported by the ANACOGA research network. 
where $\hat{\rho}_{Z}=\left(\partial \hat{\rho} / \partial Z_{j}^{\prime}\right)_{1 \leq j \leq \hat{N}}$ in some local coordinate system $Z^{\prime}$ near $\hat{p}$; we use here standard multi-index notation $L^{\bar{J}}:=L_{\overline{1}}^{\bar{J}_{1}} \ldots L_{\bar{n}}^{\bar{J}_{n}}$ and $|J|=J_{1}+\ldots+J_{n}$. One can show (cf. [9]) that the increasing sequence of numbers $d_{k}(q):=\operatorname{dim} E_{k}(q)$ is independent of the choice of local defining function $\hat{\rho}$ and coordinates $Z^{\prime}$, as well as of the choice of basis of the CR vector fields $L_{\overline{1}}, \ldots, L_{\bar{n}}$. We shall say, again following Lamel (loc. cit.) that $f:(M, q) \rightarrow \hat{M}$ is $\left(k_{0}, s\right)$-degenerate at $q$ if $s$ is the minimum of the decreasing sequence of codimensions of $E_{k}$, i.e.

$$
s:=\min _{k}\left(\hat{N}-d_{k}\right)
$$

and $k_{0}$ is the smallest integer such that this minimum is attained. The case where $s=0$ is actually a nondegenerate situation, and we say that $f$ is $k_{0}$-nondegenerate if it is $\left(k_{0}, 0\right)$-degenerate. If $f$ is $\left(k_{0}, s\right)$ degenerate at a point $q$ and the degeneracy $s$ is constant in a nieghborhood of $q$, then we shall say that $f$ is constantly $\left(k_{0}, s\right)$-degenerate at $q$. The notion of $\left(k_{0}, s\right)$-degeneracy will be presented in a more intrinsic form in section 3.2 .

The following are the main results in this paper.

Theorem 1. Let $M \subset \mathbb{C}^{N}$ and $\hat{M} \subset \mathbb{C}^{N+1}$ be smooth hypersurfaces which are Levi-nondegenerate at $p_{0} \in M$ and $\hat{p}_{0} \in \hat{M}$, and $f: M \rightarrow \hat{M}$ a smooth $C R$ mapping with $f\left(p_{0}\right)=\hat{p}_{0}$ which is constantly $\left(k_{0}, s\right)$-degenerate and transversal at $p_{0}$. Then $f$ is uniquely determined by its $2 k_{0}$-jet $j_{p_{0}}^{2 k_{0}} f$ at $p_{0}$ in a neighbourhood of $p_{0}$. That is, there exists a neighbourhood $U$ of $p_{0}$ in $M$ such that if $g: M \rightarrow \hat{M}$ is another smooth CR map of constant degeneracy s with $j_{p_{0}}^{2 k_{0}} f=j_{p_{0}}^{2 k_{0}} g$, then $\left.f\right|_{U}=\left.g\right|_{U}$.

Theorem 2. Let $M \subset \mathbb{C}^{N}$ and $\hat{M} \subset \mathbb{C}^{\hat{N}}$ be smooth hypersurfaces, $p_{0} \in M, \hat{p}_{0} \in \hat{M}$, $\hat{M}$ strictly pseudoconvex at $\hat{p}_{0}$, and $f: M \rightarrow \hat{M}$ a smooth CR mapping with $f\left(p_{0}\right)=\hat{p}_{0}$ which is constantly $\left(k_{0}, s\right)$ degenerate at $p_{0}$. Then $f$ is uniquely determined by its $2 k_{0}$-jet $j_{p_{0}}^{2 k_{0}} f$ at $p_{0}$ in a neighbourhood of $p_{0}$. That is, there exists a neighbourhood $U$ of $p_{0}$ in $M$ such that if $g: M \rightarrow \hat{M}$ is another smooth $C R$ map of constant degeneracy s with $j_{p_{0}}^{2 k_{0}} f=j_{p_{0}}^{2 k_{0}} g$, then $\left.f\right|_{U}=\left.g\right|_{U}$.

Let us note here that in the setting of Theorem 2, the mapping $f$ is automatically transversal (see e.g. the paper [6] by Forstneric). The reader should also notice that in Theorem 2, where the hypersurfaces are required to be strictly pseudoconvex, there is no restriction on the codimension $\hat{N}-N$ of the mapping $f$ whereas in Theorem 1, where no pseudoconvexity is assumed, the codimension is restricted to be one. The reason for this is easily seen by considering the case where $M \subset \mathbb{C}^{N}$ is given by the equation

$$
\Im z_{1}=\sum_{k=2}^{N}\left|z_{k}\right|^{2}
$$

and $\hat{M} \subset \mathbb{C}^{N+2}$ by

$$
\Im z_{1}=\sum_{k=2}^{N}\left|z_{k}\right|^{2}+\left|z_{N+1}\right|^{2}-\left|z_{N+2}\right|^{2} .
$$

The mapping

$$
\left(z_{1}, \ldots, z_{N}\right) \mapsto\left(z_{1}, \ldots, z_{N}, f(z),-f(z)\right),
$$

where $f$ is an arbitrary holomorphic function with $f(0)=0$, sends $(M, 0)$ into $(\hat{M}, 0)$. It is not difficult to see that if $k_{0}$ is the smallest integer $\geq 2$ for which there is a multi-index $\alpha \in \mathbb{Z}_{+}^{N-1}$ with $\partial_{z^{\prime}}^{\alpha} f(0) \neq 0$, where $z^{\prime}=\left(z_{2}, \ldots, z_{N}\right)$, then the mapping (3) is constantly $\left(k_{0}, 1\right)$-degenerate at 0 . Clearly, you can find two different such functions $f_{1}, f_{2}$ whose jets at 0 agree up to arbitrary order and, hence, Theorem 1 is false in codimension 2 . 
We shall see that in fact, given a mapping $f$ as in the preceding theorems, there exists a dense, open subset $M_{f}$ of $M$ such that for each $p \in M_{f}, f$ is constantly $\left(k_{0}, s\right)$ - degenerate for some $s$ and furthermore, that $k_{0} \leq \hat{N}-N+1-s \leq \hat{N}-N+1$ (see Lemma 12). Hence, we have the following corollaries.

Corollary 3. Let $M \subset \mathbb{C}^{N}$ and $\hat{M} \subset \mathbb{C}^{N+1}$ be smooth hypersurfaces which are Levi-nondegenerate, and $f: M \rightarrow \hat{M}$ and $g: M \rightarrow \hat{M}$ smooth, transversal $C R$ mappings. If for any $p_{0}$ in the dense open subset $M_{f} \cap M_{g}$ of $M$ we have that $j_{p_{0}}^{2(\hat{N}-N+1)} f=j_{p_{0}}^{2(\hat{N}-N+1)} g$, then $f=g$.

Corollary 4. Let $M \subset \mathbb{C}^{N}$ and $\hat{M} \subset \mathbb{C}^{\hat{N}}$ be smooth strictly pseudoconvex hypersurfaces and $f: M \rightarrow \hat{M}$ and $g: M \rightarrow \hat{M}$ smooth $C R$ mappings. If for any $p_{0}$ in the dense open subset $M_{f} \cap M_{g}$ of $M$ we have that $j_{p_{0}}^{2(\hat{N}-N+1)} f=j_{p_{0}}^{2(\hat{N}-N+1)} g$, then $f=g$.

As an application of Theorem \&, we obtain the following reflection principle.

Corollary 5. Let $M \subset \mathbb{C}^{N}$ and $\hat{M} \subset \mathbb{C}^{N+1}$ be real-analytic hypersurfaces which are Levi-nondegenerate, and $f: M \rightarrow \hat{M}$ a smooth transversal $C R$ mapping. Then there exists an open dense subset $M_{0} \subset M$ such that for any $p \in M_{0}$, there exists an open neighbourhood $V$ of $p$ in $\mathbb{C}^{N}$ and a holomorphic map $F: V \rightarrow \mathbb{C}^{\hat{N}}$ such that $\left.F\right|_{V \cap M}=f$.

Let us give the (short) proof here. We first point out that given a mapping $f$ as in Corollary 5 , there exists a dense, open subset $M_{0}$ of $M$ such that for each $p \in M_{0}, f$ is constantly $\left(k_{0}, s\right)$-degenerate, for some $s$, in an open neighborhood of $p$ (see Lemma 11); in fact, one may choose $M_{0}$ such that $f$ is locally constantly $\left(k_{0}, s\right)$-degenerate on $M_{0}$ with $s \leq \hat{N}-N$ and $k_{0} \leq \hat{N}-N+1-s \leq \hat{N}-N+1$ (see Lemma 12). For points $p_{0} \in M_{0}$, we can use a result of the second author ([8], Theorem 6) to conclude that the formal Taylor series of $f$ at $p_{0}$ converges (in some neighbourhood $\mathrm{V}$ of $p_{0}$ ) to a holomorphic mapping $F: V \rightarrow \mathbb{C}^{\hat{N}}$ with $F(M \cap V) \subset \hat{M}$. By Theorem 1, $\left.F\right|_{M}=f$, which proves the corollary.

The same argument (using Lemma 11 and Theorem 21) also gives an independent proof of the following theorem due to Forstneric [6].

Corollary 6. Let $M \subset \mathbb{C}^{N}$ and $\hat{M} \subset \mathbb{C}^{N+1}$ be real-analytic strictly pseudoconvex hypersurfaces and $f: M \rightarrow \hat{M}$ a smooth $C R$ mapping. Then there exists an open dense subset $M_{0} \subset M$ such that for any $p \in M_{0}$, there exists an open neighbourhood $V$ of $p$ in $\mathbb{C}^{N}$ and a holomorphic map $F: V \rightarrow \mathbb{C}^{\hat{N}}$ such that $\left.F\right|_{V \cap M}=f$.

A special case for which we also derive finite determination results without any conditions on $\hat{N}$ is the case of nondegenerate mappings. These have already been investigated by Kim and Zaitsev [10] in the general codimension case. However, our method is a bit different, and we have an easy, independent proof of the following result for nondegenerate mappings between hypersurfaces.

Theorem 7. Let $M \subset \mathbb{C}^{N}$ and $\hat{M} \subset \mathbb{C}^{\hat{N}}$ be smooth hypersurfaces, $p_{0} \in M$ a point of finite type, and let $f: M \rightarrow \hat{M}$ be a smooth $C R$ mapping which is $k_{0}$-nondegenerate at $p_{0}$. Then $f$ is uniquely determined by its $2 k_{0}$-jet $j_{p_{0}}^{2 k_{0}} f$ at $p_{0}$ in a neighbourhood of $p_{0}$. That is, there exists a neighbourhood $U$ of $p_{0}$ in $M$ such that if $g: M \rightarrow \hat{M}$ is a smooth CR map with $j_{p_{0}}^{2 k_{0}} f=j_{p_{0}}^{2 k_{0}} g$, then $\left.f\right|_{U}=\left.g\right|_{U}$.

In order to prove Theorems 1, 2, and 7 we will construct complete systems of differential equations for all the cases considered. To formulate the result more precisely, we shall use the following notation. For an open subset $U \subset M$, we shall denote by $J^{m}(U, \hat{M})$ the space of $m$-jets of smooth (not necessarily CR) mappings $F: U \rightarrow \hat{M}$ and by $j_{p}^{m} F$ the $m$-jet of such a mapping at $p \in U$ (which we shall think of as the collection of all derivatives of $F$, in some local coordinate system near $p$, up to order $m$ ). The result needed to prove Theorems 1 and 2 can now be formulated as follows. 
Theorem 8. Let $M \subset \mathbb{C}^{N}, \hat{M} \subset \mathbb{C}^{\hat{N}}$ be smooth hypersurfaces, $p_{0} \in M, \hat{p}_{0} \in \hat{M}, f: M \rightarrow \hat{M} a$ transversal smooth $C R$ map with $f\left(p_{0}\right)=\hat{p}_{0}$ of constant degeneracy $\left(k_{0}, s\right)$ at $p_{0}$, and assume that either $M \subset \mathbb{C}^{N}$ and $\hat{M} \subset \mathbb{C}^{N+1}$ are Levi nondegenerate at $p_{0}$ and $\hat{p}_{0}$ respectively, or that $M$ and $\hat{M}$ are strictly pseudoconvex at $p_{0}$ and $\hat{p}_{0}$ respectively (with no restrictions on $\hat{N}$ ). Then there exists a neighbourhood $U$ of $p_{0}$ in $M$ and a smooth function $\phi: U \times J^{2 k_{0}+2}(U, \hat{M}) \rightarrow J^{2 k_{0}+2}(U, \hat{M})$ which only depends on $M$ and $\hat{M}$ such that

$$
j_{x}^{2 k_{0}+3} f=\phi\left(x, j_{x}^{2 k_{0}+2} f\right), \quad x \in U .
$$

Furthermore, there exists a neigbourhood $V$ of $j_{p_{0}}^{2 k_{0}+2} f$ such that if $g: M \rightarrow \hat{M}$ a transversal smooth CR map with $g\left(p_{0}\right)=\hat{p}_{0}$ of constant degeneracy $\left(k_{0}, s\right)$ at $p_{0}$ with $j_{p_{0}}^{2 k_{0}+2} g \in V$, then (for some neighbourhood $U^{\prime}$ of $\left.p_{0}\right)$

$$
j_{x}^{2 k_{0}+3} g=\phi\left(x, j_{x}^{2 k_{0}+2} g\right), \quad x \in U^{\prime} .
$$

Theorems 1 and 2 follow from Theorem 8 using standard arguments about uniqueness of solutions of complete systems of differential equations (see e.g. [4]) and the fact that the $m$-jet of any mapping $f: M \rightarrow \hat{M}$, as in Theorems 1 and 2, at $p_{0}$, for any $m$ (and hence in particular the $2 k_{0}+2$-jet) is determined by its $2 k_{0}$ jet at $p_{0}$ ([8], Theorem 8 ). Thus, to prove Theorems 1 and 2 it suffices to prove Theorem 8. Also, by a remark given earlier, we could drop the assumption of transversality in the strictly pseudoconvex case, since it is satisfied automatically.

The organization of the paper is as follows: In section 2, we review the definition of a CR vector bundle, give definitions of the nondegeneracy conditions introduced above in this context, and introduce some essential notation and tools. Following that, we give the proof of Theorem 7. In the next two sections, we derive some jet reflection identities which we use in section 7 to prove Theorem 8 .

\section{CR VECTOR BUNDLES}

In this section, we define the notion of a $\mathrm{CR}$ vector bundle. This notion is not new and has been extensively used in the literature, but we give a thorough definition nonetheless. We also give some examples, and introduce the canonical derivative operator $D$ (which is the same as $\bar{\partial}_{b}$ in most of the literature). For our purposes, the local formalism of this derivative operator is more important than the global formalism developed alongside it.

2.1. Definition. Let $M$ be a CR manifold. Let us first recall some basic definitions. We are given a subbundle $\mathcal{V}(M) \subset \mathbb{C} T M$ (which we will refer to as the $C R$ bundle of $M$ ) which satisfies

$$
[\mathcal{V}(M), \mathcal{V}(M)] \subset \mathcal{V}(M), \quad \text { and } \mathcal{V}(M) \cap \overline{\mathcal{V}(M)}=\{0\}
$$

In (6), $[\mathcal{V}(M), \mathcal{V}(M)] \subset \mathcal{V}(M)$ means that for all sections $X, Y$ of $\mathcal{V}(M)$, the section $[X, Y]$ of $\mathbb{C} T M$ takes values in $\mathcal{V}(M)$, and 0 stands for the 0 section of $\mathbb{C} T M$. If $M$ is a CR manifold, we also say that $M$ has a CR structure, and sometimes we even refer to $\mathcal{V}(M)$ as a CR structure on $M$. If $U \subset M$ is open, a section $\bar{L} \in \Gamma(U, \mathcal{V}(M))$ is called a CR vector field over $U$. A CR function on $U$ is one which is annihilated by all $\mathrm{CR}$ vector fields on $U$. If $M, \hat{M}$ are two CR manifolds and $f: M \rightarrow \hat{M}$ is a map, say of class $C^{1}$, we say that $f$ is CR provided $f_{*}(\mathcal{V}(M)) \subset \mathcal{V}(\hat{M})$. Let us also recall that if $X$ is a complex manifold, then $X$ has a natural CR structure given by $\mathcal{V}(X)=T^{(0,1)} X$.

We consider a complex vector bundle $E$ over $M$ with projection $\pi: E \rightarrow M$. A vector bundle atlas $\mathcal{A}$ consists of pairs $\left(U_{\alpha}, \phi_{\alpha}\right)$ where $\left\{U_{\alpha}: \alpha \in A\right\}$ is an open cover of $M$, and $\phi_{\alpha}: \pi^{-1}\left(U_{\alpha}\right) \rightarrow U_{\alpha} \times \mathbb{C}^{k}$ is a diffeomorphism such that $\left.\pi\right|_{\pi^{-1}\left(U_{\alpha}\right)}=\operatorname{proj}_{1} \circ \phi_{\alpha}$, where $\operatorname{proj}_{l}$ denotes the projection on the $l$ th component in a product. The chart change $\phi_{\alpha} \circ \phi_{\beta}^{-1}:\left(U_{\alpha} \cap U_{\beta}\right) \times \mathbb{C}^{k} \rightarrow\left(U_{\alpha} \cap U_{\beta}\right) \times \mathbb{C}^{k}$, is of the form 
$\phi_{\alpha} \circ \phi_{\beta}^{-1}(x, v)=\left(x, \phi_{\alpha \beta}(x) v\right)$, where the transition functions $\phi_{\alpha \beta}$ are smooth functions (on $\left.U_{\alpha} \cap U_{\beta}\right)$ valued in $G L\left(\mathbb{C}^{k}\right)$ satisfying the usual cocyle conditions (cf. e.g []]).

We say that $(E, \mathcal{A})$ is a $C R$ vector bundle if for $\alpha, \beta \in A$ the transition functions $\phi_{\alpha \beta}$ are CR. We refer to $\mathcal{A}$ as a $C R$ atlas for $E$, and if a CR atlas is fixed, we simply say that $E$ is a CR vector bundle.

A vector bundle chart $(U, \phi)$ for $E$ is said to be compatible with $\mathcal{A}$ if $\phi_{\alpha} \circ \phi^{-1}(x, v)=\left(x, \psi_{\alpha}(x) v\right)$ where $\psi_{\alpha}: U \cap U_{\alpha} \rightarrow G L\left(\mathbb{C}^{k}\right)$ is $\mathrm{CR}$ for all $\alpha \in A$; we say that $(U, \phi)$ is a $C R \operatorname{chart}($ for $(E, \mathcal{A})$ ), and that $U$ is a trivializing neighbourhood. If $\mathcal{A}$ has the property that every $\mathrm{CR}$ chart $(U, \phi)$ for $(E, \mathcal{A})$ belongs to $\mathcal{A}$, we say that $\mathcal{A}$ is a maximal $C R$ atlas (for E). By including all compatible charts we can associate to $\mathcal{A}$ a unique maximal atlas $\mathcal{A}_{\text {max }}$ with the property that $\mathcal{A} \subset \mathcal{A}_{\max }$. Two CR vector bundles $(E, \mathcal{A})$ and $\left(E, \mathcal{A}^{\prime}\right)$ are compatible if $\mathcal{A}_{\max }=\mathcal{A}_{\text {max }}^{\prime}$. Equivalently, the $\mathrm{CR}$ vector bundles $(E, \mathcal{A})$ and $\left(E, \mathcal{A}^{\prime}\right)$ are compatible if every chart $\left(U_{\alpha}, \phi_{\alpha}\right) \in \mathcal{A}$ is a CR chart for $\left(E, \mathcal{A}^{\prime}\right)$ and vice versa. If a CR atlas $\mathcal{A}$ for $E$ is fixed, we drop the atlas from the notation, understanding that if we refer to $E$ we really refer to $(E, \mathcal{A})$.

We should point out that a given vector bundle $E$ may have many incompatible $\mathrm{CR}$ vector bundle structures. For instance, the trivial line bundle $E=M \times \mathbb{C}$ with the trivial $\mathrm{CR}$ atlas $\mathcal{A}$ consisting of the single chart $(M, \phi)$ with $\phi(x, u)=(x, u)$ is compatible with the $\mathrm{CR}$ atlas $\mathcal{A}^{\prime}$ consisting of the single chart $\left(M, \phi^{\prime}\right)$ with $\phi^{\prime}(x, u)=(x, f(x) u)$, where $f: M \rightarrow \mathbb{C}$ is some nonvanishing function, if and only if $f$ is CR.

2.2. The canonical $\mathbf{C R}$ structure. In what follows, $(E, \mathcal{A})$ will denote a $\mathrm{CR}$ vector bundle over the $\mathrm{CR}$ manifold $M$. We shall keep the $\mathrm{CR}$ atlas $\mathcal{A}$ fixed and simply refer to the $\mathrm{CR}$ vector bundle $(E, \mathcal{A})$ as $E$. In this section, we shall define a CR structure on $E$. The main point is that the CR structures induced on $\pi^{-1}\left(U_{\alpha}\right)$ by the product $\mathrm{CR}$ structures on $U_{\alpha} \times \mathbb{C}^{k}$, via the diffeomorphisms $\phi_{\alpha}$, fit together since the transition functions are CR. The CR structure induced on $E$ in this way can also be characterized by a property of sections of $E$.

Lemma 9. Assume that at each point $p \in M$ there exists a $C R$ function $g$ with $\bar{L} \bar{g}(p) \neq 0$ for some $C R$ vector field $\bar{L}$. Then there exists a unique $C R$ structure on $E$ such that a section $\sigma \in \Gamma(U, E)$ on an open subset $U \subset M$ is $C R$ if and only if for each $C R$ chart $(V, \phi)$ of $E$ the components of $\operatorname{proj}_{2} \circ \phi \circ \sigma$ are $C R$ functions on $U \cap V$. Furthermore, relative to this $C R$ structure on $E$, the projection $\pi$ is $C R$, and for each $p \in M$, there exist a neighbourhood $U$ of $p$ and a local basis of CR sections for $E$ over $U$.

Before we start with the proof, let us explain some of the notions used in the lemma. $M$ is of finite type if the Lie algebra generated by the CR vector fields and their conjugates, evaluated at $p \in M$, is all of $\mathbb{C} T_{p} M$. A CR section on $U$ is a section $\sigma \in \Gamma(U, E)$ which is $\mathrm{CR}$ as a map $U \rightarrow E$, i.e. $\sigma_{*} \mathcal{V}(M)_{p} \subset \mathcal{V}(E)_{\sigma(p)}$ for all $p \in U$. A local basis of CR sections (on $U$ ) is a collection of CR sections $\sigma_{1}, \ldots, \sigma_{k} \in \Gamma(U, E)$ such that $\sigma_{1}(p), \ldots, \sigma_{k}(p)$ is a basis of $E_{p}$ for $p \in U$.

We will use that a bundle chart $\left(U_{\alpha}, \phi_{\alpha}\right)$ induces a splitting of the tangent space $T_{P} E$ for $P \in \pi^{-1}\left(U_{\alpha}\right)$ : If we write $\phi_{\alpha}(P)=(p, v)$, then $\mathbb{C} T_{P} E \cong \mathbb{C} T_{(p, v)}\left(U_{\alpha} \times \mathbb{C}^{k}\right) \cong \mathbb{C} T_{p} M \oplus \mathbb{C} T_{v} \mathbb{C}^{k}$. The former isomorphism is given by the push forward $\left(\phi_{\alpha}\right)_{*}$. To make the latter isomorphism explicit, let $\iota_{1}$ denote the natural inclusion $\mathbb{C} T_{p} M \hookrightarrow \mathbb{C} T_{p} M \oplus \mathbb{C} T_{v} \mathbb{C}^{k}$ given by $\iota_{1}(X)=X \oplus 0$, and similarly $\iota_{2}: \mathbb{C} T_{v} \mathbb{C}^{k} \hookrightarrow \mathbb{C} T_{p} M \oplus \mathbb{C} T_{v} \mathbb{C}^{k}$. A tangent vector $\iota_{1} X+\iota_{2} Y \in \mathbb{C} T_{p} M \oplus T_{v} \mathbb{C}^{k}$ with $X \in \mathbb{C} T_{p_{0}} M$ and $Y \in \mathbb{C} T_{v_{0}} \mathbb{C}^{k}$ acts on the germ of a smooth function $f(p, v)$ at $\left(p_{0}, v_{0}\right)$ by $\left(\iota_{1} X+\iota_{2} Y\right) f=X\left(f\left(p, v_{0}\right)\right)+Y\left(f\left(p_{0}, v\right)\right)$.

Proof of Lemma 9. We define $\mathcal{V}(E)_{P}=\left(\phi_{\alpha}^{-1}\right)_{*}\left(\mathcal{V}(M)_{p} \oplus T_{v}^{(0,1)} \mathbb{C}^{k}\right)$. We will show that $\mathcal{V}(E)$ defined this way is the unique CR structure claimed in Lemma 9. To start, let us first show that this definition is independent of the chart used. Consider a chart change $\phi=\phi_{\alpha} \circ \phi_{\beta}^{-1}$. We denote the fiber coordinate in the chart $\phi_{\alpha}$ by $u=\left(u^{1}, \ldots, u^{k}\right)$, the fiber coordinate in the chart $\phi_{\beta}$ by $v=\left(v^{1}, \ldots, v^{k}\right)$, and let $P_{0} \in \pi^{-1}\left(U_{\alpha} \cap U_{\beta}\right), \phi_{\alpha}\left(P_{0}\right)=\left(p_{0}, u_{0}\right), \phi_{\beta}\left(P_{0}\right)=\left(p_{0}, v_{0}\right)$; thus,

$$
(p, u)=\phi(p, v)=\left(p, \phi_{\alpha \beta}(p) v\right) .
$$


We must show that $\phi_{*}\left(\mathcal{V}(M)_{p_{0}} \oplus T_{v_{0}}^{(0,1)} \mathbb{C}^{k}\right)=\mathcal{V}(M)_{p_{0}} \oplus T_{v_{0}}^{(0,1)} \mathbb{C}^{k}$, or equivalently $\phi_{*}\left(\iota_{1} \mathcal{V}(M)_{p_{0}}\right) \subset$ $\mathcal{V}(M)_{p} \oplus T_{v_{0}}^{(0,1)} \mathbb{C}^{k}$ and $\phi_{*}\left(\iota_{2} T_{v_{0}}^{(0,1)} \mathbb{C}^{k}\right) \subset \mathcal{V}(M)_{p_{0}} \oplus T_{v_{0}}^{(0,1)} \mathbb{C}^{k}$. For this purpose, let $\bar{L}_{p_{0}} \in \mathcal{V}(M)_{p_{0}}$. Then

$$
\begin{aligned}
\left(\phi_{*} \iota_{1} \bar{L}_{p_{0}}\right) f(p, u) & =\bar{L}_{p_{0}} f\left(p, \phi_{\alpha \beta}(p) v_{0}\right) \\
& =\bar{L}_{p_{0}} f\left(p, \phi_{\alpha \beta}\left(p_{0}\right) v_{0}\right)+\frac{\partial f}{\partial u} \bar{L}_{p_{0}}\left(\phi_{\alpha \beta}(p)\right) v_{0}+\frac{\partial f}{\partial \bar{u}} \bar{L}_{p_{0}}\left(\overline{\phi_{\alpha \beta}(p)}\right) \bar{v}_{0} \\
& =\iota_{1} \bar{L}_{p_{0}} f+\left(\left.\iota_{2} \frac{\partial}{\partial \bar{u}}\right|_{u_{0}} f\right) \bar{L}_{p_{0}}\left(\overline{\phi_{\alpha \beta}(p)}\right) \bar{v}_{0}
\end{aligned}
$$

the last equality holds since $\phi_{\alpha \beta}$ is CR. Also,

$$
\begin{aligned}
\left(\left.\phi_{*} \iota_{2} \frac{\partial}{\partial \bar{v}^{j}}\right|_{v_{0}}\right) f(p, u) & =\left.\iota_{2} \frac{\partial}{\partial \bar{v}^{j}}\right|_{v_{0}} f\left(p, \phi_{\alpha \beta}(p) v\right) \\
& =\left.\frac{\partial}{\partial \bar{v}^{j}}\right|_{v_{0}} f\left(p_{0}, \phi_{\alpha \beta}\left(p_{0}\right) v\right) \\
& =\left(\left.\iota_{2} \frac{\partial}{\partial \bar{u}}\right|_{u_{0}} f\right) \phi_{\alpha \beta}\left(p_{0}\right),
\end{aligned}
$$

so that $\phi_{*} \iota_{1} \bar{L}_{p_{0}} \in \mathcal{V}(M)_{p_{0}} \oplus T_{u_{0}}^{(0,1)} \mathbb{C}^{k}$ and $\left.\phi_{*} \iota_{2} \frac{\partial}{\partial \bar{v}^{j}}\right|_{v_{0}} \in T_{u_{0}}^{(0,1)} \mathbb{C}^{k}$. Hence $\phi_{*}\left(\mathcal{V}(M)_{p_{0}} \oplus T_{v_{0}}^{(0,1)} \mathbb{C}^{k}\right)=$ $\mathcal{V}(M)_{p_{0}} \oplus T_{u_{0}}^{(0,1)} \mathbb{C}^{k}$, as claimed.

It is easy to check that $[\mathcal{V}(E), \mathcal{V}(E)] \subset \mathcal{V}(E)$ (since the derivatives in the fiber directions and the derivatives in the base directions commute). It also follows immediately that $\mathcal{V}(E) \cap \overline{\mathcal{V}(E)}=\{0\}$. We conclude that $\mathcal{V}(E)$ defines a CR structure on $E$; furthermore, $\pi_{*}(\mathcal{V}(E))=\mathcal{V}(M)$, so that $\pi$ is CR as claimed.

We next verify that $\mathcal{V}(E)$ has the properties claimed in Lemma 9. Let $\sigma:\left.U \rightarrow E\right|_{U}$ be a section, and $(V, \phi)$ a CR chart with $U \cap V$ nonempty. By the construction of the CR structure on $E, \sigma$ is CR if and only if $\phi \circ \sigma$ is CR. Since $\sigma$ is a section, we have $\phi \circ \sigma(p)=(p, \tilde{\sigma}(p))$, where $\tilde{\sigma}=\operatorname{proj}_{2} \circ \phi \circ \sigma=\left(\sigma^{1}, \ldots, \sigma^{k}\right)^{t}$. We compute $(\phi \circ \sigma)_{*} \bar{L}_{p_{0}}$, where, for ease of notation, we drop the inclusions $\iota_{1}, \iota_{2}$ :

$$
\begin{aligned}
\left((\phi \circ \sigma)_{*} \bar{L}_{p_{0}}\right) f(p, u) & =\bar{L}_{p_{0}} f(p, \tilde{\sigma}(p)) \\
& =\bar{L}_{p_{0}} f\left(p, \tilde{\sigma}\left(p_{0}\right)\right)+\left.\frac{\partial f\left(p_{0}, u\right)}{\partial \bar{u}}\right|_{\tilde{\sigma}\left(p_{0}\right)} \bar{L}_{p_{0}} \overline{\tilde{\sigma}}+\left.\frac{\partial f\left(p_{0}, u\right)}{\partial u}\right|_{\tilde{\sigma}\left(p_{0}\right)} \bar{L}_{p_{0}} \tilde{\sigma} .
\end{aligned}
$$

From this we see that $\phi \circ \sigma$ (and hence $\sigma$ ) is CR if and only if the components of $\tilde{\sigma}$ are CR functions.

Now let $p \in M$ and assume that there exists a CR function $g$ with the property that $\bar{L} \bar{g}(p) \neq 0$. Let us show that the CR structure constructed above is unique with the properties in Lemma 9. Pick $P_{0} \in E$ and a CR chart $(V, \phi)$ with $P_{0} \in \pi^{-1}(V)$. Write $\phi\left(P_{0}\right)=\left(p_{0}, u_{0}\right)$. There is no loss of generality in assuming that $g\left(p_{0}\right)=u_{0}$. Using the computation above, first with $\tilde{\sigma}=u_{0}$ and then with $\tilde{\sigma}=g e_{j}$ for standard unit vectors $e_{j}, j=1, \ldots, k$, we conclude, since $\sigma=\phi^{-1}(p, \tilde{\sigma})$ is CR for each of the choices of $\tilde{\sigma}$ above, that $\mathcal{V}(E)$ must be the bundle $\left(\phi^{-1}\right)_{*}\left(\mathcal{V}(M)_{p_{0}} \oplus T_{u_{0}}^{(0,1)} \mathbb{C}^{k}\right)$. This completes the proof of the Lemma.

Remark 1. In what follows, unless specifically stated otherwise, every CR vector bundle will be equipped with the canonical CR structure provided by Lemma 9 . 
2.3. Constructions with CR vector bundles. All standard vector bundle constructions work in the category of CR vector bundles. In particular, the sum, tensor product, and exterior product of CR vector bundles carry the structure of a $\mathrm{CR}$ vector bundle, and the dual bundle $E^{*}$ is a CR vector bundle. Also, if $\hat{E}$ is a $\mathrm{CR}$ vector bundle over another CR manifold $\hat{M}$, and $f: M \rightarrow \hat{M}$ is a CR map, the pullback bundle $f^{\sharp} \hat{E}$ inherits the structure of a CR vector bundle.

Let us discuss the construction of the pullback bundle in detail. We let $E_{x}=\hat{E}_{f(x)}$ and define a vector bundle structure on $E=\cup_{x \in M} E_{x}$ as follows: If $\hat{U} \subset \hat{M}$ is open and $\hat{\phi}:(\hat{\pi})^{-1}(\hat{U}) \rightarrow \hat{U} \times \mathbb{C}^{k}$ is a CR chart for $\hat{E}$ over $\hat{U},\left(\hat{\phi}^{-1} \circ(f \times \mathrm{id})\right)^{-1}$ is a bundle chart for $E$ over $U=f^{-1}(\hat{U})$. Since the transition functions for $E$ are just the pullbacks (by $f$ ) of the transition functions for $\hat{E}, E$ is a CR vector bundle. The map $f: M \rightarrow \hat{M}$ lifts to a differentiable vector bundle map $F: E \rightarrow \hat{E}$. The canonical CR structure on $\hat{E}$ is connected with the structure on $E$ by $\mathcal{V}(E)_{p} \subset \mathcal{V}(\hat{E})_{f(p)}$, so that $F: E \rightarrow \hat{E}$ is a CR map. Also note that the pullback (by $f) f^{\sharp} \sigma \in \Gamma\left(f^{-1}(\hat{U}), E\right)$ of a CR section $\sigma \in \Gamma(\hat{U}, \hat{E})$ is a CR section of $E$.

2.4. The derivative operator. In analogy with complex analytic vector bundles, we can differentiate sections of a $C R$ vector bundle $E$ (equipped with the canonical CR structure provided by Lemma 9) with respect to $\mathrm{CR}$ vector fields. To see this, let $\sigma_{1}, \ldots, \sigma_{k}$ be a local basis of $\mathrm{CR}$ sections over some open set $U$. If $\sigma \in \Gamma(U, E)$ is given by $\sigma=\sum_{j=1}^{k} a^{j} \sigma_{j}$ with $a^{j} \in C^{\infty}(U)$ and $\bar{L}$ is a CR vector field on $U$, we define

$$
D_{\bar{L}} \sigma=\sum_{j=1}^{k}\left(\bar{L} a^{j}\right) \sigma_{j}
$$

This definition is invariant under changes of the local CR basis $\sigma_{j}$ : Observe that a section $\sigma \in \Gamma(U, E)$, $\sigma=\sum_{j=1}^{k} a^{j} \sigma_{j}$ is $\mathrm{CR}$ if and only if all the $a^{j}$ are $\mathrm{CR}$ functions on $U$. Hence, if $\tilde{\sigma}_{1}, \ldots, \tilde{\sigma}_{k}$ is another basis of CR sections, $\sigma_{j}=\sum_{l} c_{j}{ }^{l} \tilde{\sigma}_{l}$ where the $c_{j}{ }^{l}$ are CR functions on $U$. It follows that $\sigma=\sum_{l}\left(\sum_{j} c_{j}{ }^{l} a^{j}\right) \tilde{\sigma}_{l}=$ $\sum_{l} \tilde{a}^{l} \tilde{\sigma}_{l}$, and

$$
\begin{aligned}
\sum_{l}\left(\bar{L} \tilde{a}^{l}\right) \tilde{\sigma}_{l} & =\sum_{l} \bar{L}\left(\sum_{j} c_{j}{ }^{l} a^{j}\right) \tilde{\sigma}_{l} \\
& =\sum_{l}\left(\sum_{j} c_{j}{ }^{l} \bar{L} a^{j}\right) \tilde{\sigma}_{l} \\
& =\sum_{j}\left(\bar{L} a^{j}\right) \sigma_{j},
\end{aligned}
$$

so that $D_{\bar{L}} \sigma$ is independent of the local basis of CR sections used. This allows us to define $D_{\bar{L}} \sigma$ for a section $\sigma \in \Gamma(U, E)$ on any open set $U \in M$. Note that $\sigma$ is $\mathrm{CR}$ if and only if $D_{\bar{L}} \sigma=0$ (the 0 section) for all CR vector fields $\bar{L}$.

We also note that the operator $\omega \mapsto D_{\bar{L}} \omega$ is local, and since $D_{a \bar{L}} \omega=a D_{\bar{L}} \omega$ for any function $a,\left(D_{\bar{L}} \omega\right)_{p}$ depends only on $\bar{L}_{p}$. Moreover, $D$ satisfies the product rule:

$$
D_{\bar{L}}(a \omega)=(\bar{L} a) \omega+a D_{\bar{L}} \omega
$$

If $\omega=\left(\omega_{1}, \ldots, \omega_{k}\right)$ is any local (not necessarily CR) basis of sections over $U$ and $\bar{L}_{1}, \ldots, \bar{L}_{n}$ is a local basis of CR vector fields over $U$, we can write $D_{\bar{L}_{i}} \omega_{j}=\sum_{l} a_{i j}{ }^{l} \omega_{l}$, (or, using matrix notation, $D_{\bar{L}_{i}} \omega=A_{i} \omega$ ) and $A_{i}=\left(a_{i j}{ }^{l}\right)_{i}$ determines $D_{\bar{L}} \sigma$ for all sections $\sigma$ over $U$. If we change basis by $\tilde{\omega}_{j}=\sum_{m} C_{j}^{m} \omega_{m}$, which 
we will write in matrix notation as $\tilde{\omega}=C \omega$, we obtain

$$
\begin{aligned}
D_{\bar{L}_{i}} \tilde{\omega} & =D_{\bar{L}_{i}} C \omega \\
& =\left(\bar{L}_{i} C\right) \omega+C\left(D_{\bar{L}_{i}} \omega\right) \\
& =\left(\left(\bar{L}_{i} C\right) C^{-1}+C A_{i} C^{-1}\right) \tilde{\omega} .
\end{aligned}
$$

This means that under a change of basis, the $A_{i}$ transform as follows:

$$
\tilde{\omega}=C \omega \Longrightarrow \tilde{A}_{i}=\left(\bar{L}_{i} C\right) C^{-1}+C A_{i} C^{-1} .
$$

We can also think of $D$ as an operator from sections of $E$ to sections of $E \otimes \mathcal{V}(M)^{*} \simeq E \otimes\left(\mathbb{C} T^{*} M / \mathcal{V}(M)^{\perp}\right)$. Since $D$ satisfies the product rule $(\mathbb{7})$, we may consider $D$ as a partial connection in $E$.

If $E$ is the trivial bundle $M \times \mathbb{C}$ or the holomorphic cotangent bundle $T^{\prime} M=\mathcal{V}(M)^{\perp}$, then $D$ is just $\bar{\partial}_{b}$. If $M$ is a complex manifold and $E$ is an analytic vector bundle, $D$ is $\bar{\partial}$.

\section{The CR vector Bundle structure on the holomorphic Cotangent Bundle}

3.1. Definition. Let now $M$ be an integrable CR manifold of CR dimension $n=\operatorname{dim} \mathcal{V}(M)_{p}$ and $\mathrm{CR}$ codimension $d=\operatorname{dim} M-2 n$. Integrability means that at each point $p \in M$ there exist a neighbourhood $U$ of $p$ and $N=n+d$ smooth CR functions $Z_{j}$ on $U, j=1, \ldots, N$ such that the differentials $d Z_{1}, \ldots, d Z_{N}$ are linearly independent on $U$. The functions $Z_{1}, \ldots, Z_{N}$ are called a family of basic solutions in $U$. One can show that $q \mapsto\left(Z_{1}(q), \ldots, Z_{N}(q)\right)$ embeds $M$, locally near $p \in M$, as a generic submanifold in $\mathbb{C}^{N}$. Conversely, any embeddable (in this sense) CR manifold is integrable.

We recall the definition of the holomorphic cotangent bundle $T^{\prime} M$ : At each $p \in M, T_{p}^{\prime} M=\mathcal{V}(M)_{p}^{\perp}$. Since $\operatorname{dim} \mathbb{C} T_{p} M=2 n+d$ and $\operatorname{dim} \mathcal{V}(M)_{p}=n, \operatorname{dim} T_{p}^{\prime} M=N$. Hence, on a neighbourhood $U$ of a fixed point $p$ as above, the 1 -forms $d Z_{1}, \ldots, d Z_{N}$ form a basis of the sections of $T^{\prime} M$ over $U$. We want to define a CR atlas on $T^{\prime} M$ such that the bases obtained in this way are CR bases of sections. Assume that we have another family of basic solutions $\tilde{Z}_{1}, \ldots, \tilde{Z}_{N}$ on the open set $V \in M$. Then $d \tilde{Z}_{j}=\sum_{k} a_{j}^{k} d Z_{k}$ on $U \cap V$, and exterior differentiation yields

$$
\sum_{k} d a_{j}^{k} \wedge d Z_{k}=0
$$

in the overlap $U \cap V$. By Cartan's Lemma, we conclude that $d a_{j}{ }^{k}=\sum C_{k}^{l} d Z_{l}$, and hence, $\bar{L} a_{j}{ }^{k}=$ $\left\langle d a_{j}{ }^{k}, \bar{L}\right\rangle=0$ for all CR vector fields $\bar{L}$, so that the $a_{j}{ }^{k}$ are CR functions on $U \cap V$. Hence, if we define a CR chart for $T^{\prime} M$ over $U$ by $\phi\left(\omega_{p}\right)=\left(p, \lambda^{1}, \ldots, \lambda^{N}\right)$ where $\omega_{p} \in T_{p}^{\prime} M$ is written as $\omega_{p}=\sum_{j} \lambda^{j}\left(d Z_{j}\right)_{p}$, then, by the computation above, the collection of all such charts defines a CR atlas on $T^{\prime} M$. We equip $T^{\prime} M$ with the canonical CR structure, relative to this CR atlas, given by Lemma 9 .

By the same argument as above using Cartan's lemma, we see that the following lemma holds.

Lemma 10. Let $g$ be a smooth function on an open set $U \subset M$. Then $g$ is $C R$ if and only if $d g$ is a $C R$ section of $T^{\prime} M$ over $U$.

Sections of $T^{\prime} M$ are called holomorphic forms (on $M$ ). Let now $\omega \in \Gamma\left(M, T^{\prime} M\right.$ ), and let $\bar{L}$ be a CR vector field on $M$. The following useful formula holds:

$$
\left.D_{\bar{L}} \omega=\bar{L}\right\lrcorner d \omega .
$$


To verify this identity, let $X \in \Gamma(M, \mathbb{C} T M)$ be a tangent vector field, and $\omega=\sum_{j} a^{j} d Z_{j}$ locally. Then

$$
\begin{aligned}
\langle\bar{L}\lrcorner d \omega, X\rangle & =\langle d \omega, \bar{L} \wedge X\rangle \\
& =\sum_{j}\left\langle d a^{j} \wedge d Z_{j}, \bar{L} \wedge X\right\rangle \\
& =\sum_{j}\left(d a^{j}(\bar{L}) d Z_{j}(X)-d a^{j}(X) d Z_{j}(\bar{L})\right) \\
& =\sum_{j}\left(\bar{L} a^{j}\right) d Z_{j}(X) \\
& =\left\langle D_{\bar{L}} \omega, X\right\rangle .
\end{aligned}
$$

3.2. Nondegeneracy conditions. Recall that the characteristic bundle $T^{0} M$ is defined by

$$
T^{0} M=(\mathcal{V}(M) \oplus \overline{\mathcal{V}(M)})^{\perp} .
$$

A characteristic form is a real nonvanishing section of $T^{0} M$, and we write $\xi^{0}(U)$ for the space of characteristic forms on $U \subset M$. We usually denote a characteristic form by $\theta$.

By the discussion above, an integrable CR manifold $M$ is finitely nondegenerate (at $p$ ) as introduced by Baouendi, Huang and Rothschild [3] if and only if for some $k$,

$$
\operatorname{span}\left\{\left(D_{\bar{L}_{1}} \ldots D_{\bar{L}_{l}} \theta\right)(p): \bar{L}_{j} \in \Gamma(U, \mathcal{V}(M)), \theta \in \xi^{0}(U), l \leq k\right\}=T_{p}^{\prime} M,
$$

where $U$ is a suitable small neighbourhood of $p$. This follows from the characterization of finite nondegeneracy found in e.g. [2] and equation (8).

We will use the notion of the pullback bundle introduced before in order to give an intrinsic characterization of the nondegeneracy of a CR map $f: M \rightarrow \hat{M}$ as introduced in [9]. Let $E=f^{\sharp} T^{\prime} \hat{M}$. $f$ is nondegenerate at $p_{0}$ if and only if for some $k$,

$$
\operatorname{span}\left\{D_{\bar{L}_{1}} \ldots D_{\bar{L}_{k}} f^{\sharp} \theta\left(p_{0}\right): \bar{L}_{j} \in \Gamma(U, \mathcal{V}(M)), \theta \in \hat{\xi}(\hat{U}), j \leq k\right\}=E_{p_{0}},
$$

where again $U$ is some neighbourhood of $p_{0}$, contained in $f^{-1}(\hat{U})$. More generally, we can define subspaces $E_{p}^{k} \subset E_{p}$ (for $p \in U$, say) by

$$
E_{p}^{k}=\operatorname{span}\left\{D_{\bar{L}_{1}} \ldots D_{\bar{L}_{l}} \theta(p): \bar{L}_{j} \in \Gamma(U, \mathcal{V}(M)), \theta \in \xi^{\prime}(U), l \leq k\right\}
$$

Then $E_{p}^{0} \subset E_{p}^{1} \subset \cdots \subset E_{p}^{k} \subset \ldots$, and for some smallest integer $k_{0}=k_{0}(p), E_{p}^{k}=E_{p}^{k_{0}}$ for $k \geq k_{0}$. We call $s=s(p)=\operatorname{dim} E_{p}-\operatorname{dim} E_{p}^{k_{0}(p)}$ the degeneracy of $f$ at $p$ and say that $f$ is $\left(k_{0}, s\right)$-degenerate at $p$; we say that $f$ is of constant degeneracy $s$ at $p_{0}$ if $s(p) \equiv d$ is constant in a neighbourhood of $p_{0}$, and in this case we also say that $f$ is constantly $\left(k_{0}, s\right)$-degenerate (note that the constancy only applies to $s$ ). This characterization is equivalent to the definition given in [8], by a computation in coordinates which we leave to the reader.

Note that $p \mapsto s(p)$ is upper semicontinuous. Hence, we have the following:

Lemma 11. Let $f: M \rightarrow \hat{M}$ be a smooth CR map. Then there exists an open dense subset $N \subset M$ such that $f$ is of constant degeneracy at every point of $N$.

Let us actually observe some more. It is proved in 8 that the degeneracy $s$ of a transversal map is bounded by $\hat{N}-N$. Furthermore it follows from that paper (and is easy to prove), that $\operatorname{dim} E_{p}^{1}=N$ for any point $p$, if $\hat{M}$ is Levi nondegenerate and $f$ is an embedding. Now note that if on any open subset $U$ we had $E_{p}^{k+1} \subset E_{p}^{k}$ for $p \in U$, it follows that $E_{p}=E_{p}^{k}$. It follows that if $f$ is of constant degeneracy $s$ on $U$, then generically in $U, k_{0} \leq \hat{N}-N+1-s \leq \hat{N}-N+1$. We state this as a Lemma: 
Lemma 12. Let $f: M \rightarrow \hat{M}$ be a smooth transversal $C R$ embedding between Levi nondegenerate hypersurfaces $M$ and $\hat{M}$. Then there exists an open dense subset $N \subset M$ such that $f$ is of constant degeneracy $\left(k_{0}, s\right)$ at every point of $N$ for some $s \leq \hat{N}-N$ and $k_{0} \leq \hat{N}-N+1-s \leq \hat{N}-N+1$.

3.3. Some computations. From now on, unless stated otherwise, we assume that $M$ and $\hat{M}$ are of hypersurface type and integrable (so that the holomorphic cotangent bundles are CR vector bundles), and that $f: M \rightarrow \hat{M}$ is CR. Let us now fix a point $p_{0}$ (which we denote by 0 from now on) and a local basis $L_{\overline{1}}, \ldots L_{\bar{n}}$ of the CR vector fields on $M$ near 0 . We also choose a characteristic form $\theta$ for $M$ near 0 and a real vector field $T$ with $\langle\theta, T\rangle=1$. We denote by $\theta^{a}$ the dual form (relative to the basis of vector fields $\left.T, L_{1}, \ldots, L_{n}, L_{\overline{1}}, \ldots, L_{\bar{n}}\right)$ to the anti $\mathrm{CR}$ vector field $L_{a}$. We write $\hat{0}=f(0)$ and, as before, $\hat{\theta}$ denotes a (local) characteristic form on $\hat{M}$ near $\hat{0}$, and $\hat{T}$ a real vector field with $\langle\hat{\theta}, \hat{T}\rangle=1$; we extend $\hat{\theta}$ to a basis of $T^{\prime} \hat{M}$ by $\hat{n}$ forms $\hat{\theta}^{1}, \ldots, \hat{\theta}^{\hat{n}}$ which are the dual forms of a local basis $\hat{L}_{1}, \ldots \hat{L}_{\hat{n}}$ of the anti CR vector fields on $\hat{M}$. We work on a small neighbourhood of 0 which we may shrink, if necessary, without further mentioning it.

Since $f$ is CR, we can write

$$
f_{*} L_{a}=\gamma_{a}^{A} \hat{L}_{A}, \quad f_{*} L_{\bar{a}}=\overline{\gamma_{a}^{A}} \hat{L}, \quad f_{*} T=\eta^{A} \hat{L}_{A}+\bar{\eta}^{A} \hat{L}_{\bar{A}}+\xi \hat{T},
$$

or equivalently,

$$
f^{*} \hat{\theta}^{A}=\gamma_{a}^{A} \theta^{a}+\eta^{A} \theta, \quad f^{*} \hat{\theta}^{\bar{A}}=\overline{\gamma_{a}^{A}} \theta^{\bar{a}}+\overline{\eta^{A}} \theta, \quad f^{*} \hat{\theta}=\xi \theta .
$$

Here and from now on we use the summation convention; small indices $a, b$ etc. range from 1 to $n$, capital indices $A, B$ etc. from 1 to $\hat{n}$. We observe here that $f$ is transversal at $p \in M$, as defined in the introduction, if and only if $\xi(p) \neq 0$.

Let us write $D_{\bar{a}}=D_{L_{\bar{a}}}, f^{\sharp} \hat{\theta}=\tau$, and $f^{\sharp} \hat{\theta}^{A}=\tau^{A}$; thus, $\tau, \tau^{1}, \ldots, \tau^{n}$ forms a local basis (not necessarily $\mathrm{CR}$ ) for the CR vector bundle $E=f^{\sharp} T^{\prime} \hat{M}$. We define functions $g_{\bar{a}_{1} \ldots \bar{a}_{k} B}, g_{\bar{a}_{1} \ldots \bar{a}_{k}}$ on $M$ near 0 by

$$
D_{\bar{a}_{k}} D_{\bar{a}_{k-1}} \ldots D_{\bar{a}_{1}} \tau=g_{\bar{a}_{1} \ldots \bar{a}_{k} B} \tau^{B}+g_{\bar{a}_{1} \ldots \bar{a}_{k}} \tau \text {. }
$$

Note that for elements $\sigma_{p}$ of $E_{p}$, there is a natural pairing $\left\langle\sigma_{p}, X_{f(p)}\right\rangle$ with elements $X_{f(p)}$ of $\mathbb{C} T_{f(p)} \hat{M}$. With this notation, we can write

$$
g_{\bar{a}_{1} \ldots \bar{a}_{k} B}=\left\langle D_{\bar{a}_{k}} D_{\bar{a}_{k-1}} \ldots D_{\bar{a}_{1}} \tau, \hat{L}_{B}\right\rangle, \quad g_{\bar{a}_{1} \ldots \bar{a}_{k}}=\left\langle D_{\bar{a}_{k}} D_{\bar{a}_{k-1}} \ldots D_{\bar{a}_{1}} \tau, \hat{T}\right\rangle .
$$

We introduce similar notation for sections of $T^{\prime} \hat{M}$ and $T^{\prime} M$ :

$$
\begin{gathered}
D_{\bar{a}_{k}} \ldots D_{\bar{a}_{1}} \theta=h_{\bar{a}_{1} \ldots \bar{a}_{k} b} \theta^{b}+h_{\bar{a}_{1} \ldots \bar{a}_{k}} \theta, \\
D_{\bar{A}_{k}} \ldots D_{\bar{A}_{1}} \hat{\theta}=\hat{h}_{\bar{A}_{1} \ldots \bar{A}_{k} B} \hat{\theta}^{b}+\hat{h}_{\bar{A}_{1} \ldots \bar{A}_{k}} \hat{\theta} .
\end{gathered}
$$

With $\langle\cdot, \cdot\rangle$ denoting the canonical pairing between vectors and covectors,

$$
\begin{gathered}
h_{\bar{a}_{1} \ldots \bar{a}_{k} b}=\left\langle D_{\bar{a}_{k}} D_{\bar{a}_{k-1}} \ldots D_{\bar{a}_{1}} \theta, L_{b}\right\rangle, \quad h_{\bar{a}_{1} \ldots \bar{a}_{k}}=\left\langle D_{\bar{a}_{k}} D_{\bar{a}_{k-1}} \ldots D_{\bar{a}_{1}} \theta, T\right\rangle, \\
\hat{h}_{\bar{A}_{1} \ldots \bar{A}_{k} B}=\left\langle D_{\bar{A}_{k}} D_{\bar{A}_{k-1}} \ldots D_{\bar{A}_{1}} \hat{\theta}, \hat{L}_{B}\right\rangle, \quad \hat{h}_{\bar{A}_{1} \ldots \bar{A}_{k}}=\left\langle D_{\bar{A}_{k}} D_{\bar{A}_{k-1}} \ldots D_{\bar{A}_{1}} \hat{\tau}, \hat{T}\right\rangle .
\end{gathered}
$$

The $h_{\bar{a} b}$ relate to the Leviform of $M$ by

$$
h_{\bar{a} b}=\left\langle d \theta, L_{\bar{a}} \wedge L_{b}\right\rangle=-\theta\left(\left[L_{\bar{a}}, L_{b}\right]\right),
$$

and similarly for $\hat{M}$.

We need to introduce (but just for a short while) one more notation for our three bundles:

$$
\begin{aligned}
D_{\bar{a}} \theta^{b} & =R_{\bar{a} c}^{b} \theta^{c}+R_{\bar{a}}^{b} \theta, \\
D_{\bar{a}} \tau^{B} & =S_{\bar{a} C}^{B} \tau^{C}+S_{\bar{a}}^{B} \tau,
\end{aligned}
$$




$$
D_{\bar{A}} \hat{\theta}^{B}=\hat{R}_{\bar{A} C}^{B} \hat{\theta}^{C}+\hat{R}_{\bar{A}}^{B} \hat{\theta} .
$$

Lemma 13. Let $\sigma$ be a section of $T^{\prime} \hat{M}$. Then

$$
D_{\bar{a}} f^{\sharp} \sigma=\overline{\gamma_{a}^{A}} f^{\sharp} D_{\bar{A}} \sigma .
$$

In particular,

and

$$
\begin{gathered}
g_{\bar{a} B}=\overline{\gamma_{a}^{A}} \hat{h}_{\bar{A} B}, \quad g_{\bar{a}}=\overline{\gamma_{a}^{A}} \hat{h}_{\bar{A}}, \\
S_{\bar{a} C}^{B}=\overline{\gamma_{a}^{A}} \hat{R}_{\bar{A} C}^{B}, \quad S_{\bar{a}}^{C}=\overline{\gamma_{a}^{A}} \hat{R}_{\bar{A}}^{a} .
\end{gathered}
$$

Proof. Let us consider a local CR basis $\omega_{1}, \ldots \omega_{\hat{N}}$ for $T^{\prime} \hat{M}$. We write $\alpha_{j}=f^{\sharp} \omega_{j}$ for the corresponding local CR basis of $E$. Then locally $\sigma=a^{j} \omega_{j}$ for smooth functions $a^{j}$, and we have

$$
\begin{aligned}
D_{\bar{a}} f^{\sharp} \sigma & =D_{\bar{a}} f^{\sharp}\left(a^{j} \omega_{j}\right) \\
& =D_{\bar{a}}\left(a^{j} \circ f\right) \alpha_{j} \\
& =\left(L_{\bar{a}}\left(a^{j} \circ f\right)\right) \alpha_{j} \\
& =\left(\left(f_{*} L_{\bar{a}}\right) a^{j}\right) \alpha_{j} \\
& =\overline{\gamma_{a}^{A}}\left(L_{\bar{A}} a^{j} \circ f\right) \alpha_{j} \\
& =\overline{\gamma_{a}^{A}} f^{\sharp} D_{\bar{A}} \sigma .
\end{aligned}
$$

For the other assertions, we note that

$$
D_{\bar{a}} \tau=g_{\bar{a} B} \tau^{B}+g_{\bar{a}} \tau
$$

and

$$
D_{\bar{A}} \hat{\theta}=\hat{h}_{\bar{A} B} \hat{\theta}^{B}+h_{\bar{A}} \hat{\theta}
$$

Since

$$
\overline{\gamma_{a}^{A}} f^{\sharp}\left(D_{\bar{A}} \hat{\theta}\right)=\overline{\gamma_{a}^{A}} \hat{h}_{\bar{A} B} \tau^{B}+\overline{\gamma_{a}^{A}} \hat{h}_{\bar{A}} \tau,
$$

comparing coefficients we get (11). (12) follows by the same argument.

The following lemma is an immediate consequence of (7) and the definitions above:

Lemma 14. With the notations introduced above, the following hold:

$$
\begin{gathered}
g_{\bar{a}_{1} \ldots \bar{a}_{k} \bar{a} B}=L_{\bar{a}} g_{\bar{a}_{1} \ldots \bar{a}_{k} B}+g_{\bar{a}_{1} \ldots \bar{a}_{k} C} S_{\bar{a} B}^{C}+g_{\bar{a}_{1} \ldots \bar{a}_{k}} g_{\bar{a} B} \\
\hat{h}_{\bar{A}_{1} \ldots \bar{A}_{k} \bar{A} B}=L_{\bar{A}_{\bar{A}}} \hat{h}_{\bar{A}_{1} \ldots \bar{A}_{k} B}+\hat{h}_{\bar{A}_{1} \ldots \bar{A}_{k} C} \hat{R}_{\bar{A} B}^{C}+\hat{h}_{\bar{A}_{1} \ldots \bar{A}_{k}} \hat{h}_{\bar{A} B} \\
h_{\bar{a}_{1} \ldots \bar{a}_{k} \bar{a} b}=L_{\bar{a}} h_{\bar{a}_{1} \ldots \bar{a}_{k} b}+h_{\bar{a}_{1} \ldots \bar{a}_{k} c} R_{\bar{a} b}^{c}+h_{\bar{a}_{1} \ldots \bar{a}_{k}} h_{\bar{a} b} .
\end{gathered}
$$

We also record the following two lemmas for later use. We write $I=\left(i_{1} \ldots i_{l}\right)$, where $1 \leq i_{j} \leq n$, and we use the notation $L^{I}=L_{i_{1}} \ldots L_{i_{l}}$; for such an $I$, we write $|I|=l$.

Lemma 15. For each $k \in \mathbb{N}$, the two subspaces of $\mathbb{C}^{\hat{n}}$ defined by

$$
\begin{aligned}
& \operatorname{span}\left\{\left(g_{\bar{a}_{1} \ldots \bar{a}_{l} A}(p)\right)_{A}: l \leq k\right\} \\
& \operatorname{span}\left\{\left(L^{\bar{I}} g_{\bar{a} A}(p)\right)_{A}:|I| \leq k\right\}
\end{aligned}
$$

coincide. Consequently, the following are equivalent definitions for the degeneracy $d(p)$ of $f$ at $p$ defined in section 3.9: 
(i) $d(p)=\hat{n}-\max _{k} \operatorname{dim} \operatorname{span}\left\{\left(g_{\bar{a}_{1} \ldots \bar{a}_{k} A}(p)\right)_{A}: l \leq k\right\}$;

(ii) $d(p)=\hat{n}-\max _{k} \operatorname{dim} \operatorname{span}\left\{\left(L^{\bar{I}} g_{\bar{a} A}(p)\right)_{A}:|I| \leq k\right\}$;

and in each case, $k_{0}(p)$ is the minimal integer $k$ for which the maximum on the right hand side is attained.

The proof is by induction using (13).

Lemma 16. For each $a_{1}, \ldots, a_{k}, 1 \leq a_{l} \leq n$, and $B, 1 \leq B \leq \hat{n}$ there exist functions $u_{\bar{a}_{1} \ldots \bar{a}_{k} B}$ and $u_{\bar{a}_{1} \ldots \bar{a}_{k}}$ polynomial in their arguments with coefficients smooth on $M \times \hat{M}$ such that

$$
\begin{gathered}
g_{\bar{a}_{1} \ldots \bar{a}_{k} B}=u_{\bar{a}_{1} \ldots \bar{a}_{k} B}\left(\overline{L^{I} \gamma_{a}^{A}}\right), \quad|I| \leq k-1 \\
g_{\bar{a}_{1} \ldots \bar{a}_{k}}=u_{\bar{a}_{1} \ldots \bar{a}_{k}}\left(\overline{L^{I} \gamma_{a}^{A}}\right), \quad|I| \leq k-1 .
\end{gathered}
$$

Furthermore, $u_{\bar{a}_{1} \ldots \bar{a}_{k} A}$ and $u_{\bar{a}_{1} \ldots \bar{a}_{k}}$ depend only on $M$ and $\hat{M}$ (and not on the mapping $f$ ).

The equalities (16) and (17) mean the following: if the left hand side is evaluated at $p$, it equals the right hand side with the coefficients evaluated at $(p, f(p))$ and the arguments evaluated at $p$. We will encounter many such equalities, and we will always use this time-saving notation.

For the proof of Lemma 16, we recall that for $k=1, g_{\bar{a} B}=\overline{\gamma_{a}^{A}} \hat{h}_{\bar{A} B}$ and $g_{\bar{a}}=\bar{\gamma}_{a}^{A} \hat{h}_{\bar{A}}$ by Lemma 13. An induction and Lemma 14 finishes the proof of the Lemma 16; the details are left to the reader.

3.4. The pullback operation. In this section, we discuss the relation between our vector bundle $E=$ $f^{\sharp} T^{\prime} \hat{M}$ and the vector bundle $T^{\prime} M$ over $M$. Recall that we have a pairing $\langle\sigma, X\rangle$ for sections of $E$ and tangent vectors $X$ at $f(p)$. We define a map $\phi$ by

$$
\langle\phi \sigma, X\rangle=\left\langle\sigma, f_{*} X\right\rangle .
$$

Since $f$ is CR, $\phi$ maps sections of $E$ to sections of $T^{\prime} M$. Note that $\phi: \Gamma(U, E) \rightarrow \Gamma\left(U, T^{\prime} M\right)$ is linear over $C^{\infty}(U)$, since

$$
\langle\phi a \sigma, X\rangle=(a \sigma)\left(f_{*} X\right)=a \sigma\left(f_{*} X\right)=a\langle\phi \sigma, X\rangle .
$$

Lemma 17. For each $j, D_{\bar{j}} \phi=\phi D_{\bar{j}}$. Furthermore, $\phi \circ f^{\sharp}=f^{*}$.

Proof. Since the derivative operation is local, it is actually enough to work on a trivializing open set $U=f^{-1}(V)$, where have a family of basic solutions $\hat{Z}_{j}$ in $V$. A basis of the CR sections of $E$ is then given by $\sigma_{j}=f^{\sharp} d \hat{Z}_{j}$. Let $\tau$ be any section of $E$ over $U$. Then $\tau=a^{j} \sigma_{j}$, and $\phi \tau=a^{j} \phi\left(\sigma_{j}\right)$. By the definition of $D_{\bar{j}}$ we now see it is enough to show that if $\sigma$ is a CR section of $E$, then $\phi \sigma$ is a CR section of $T^{\prime} M$. We compute:

$$
\begin{aligned}
\left\langle\left(\phi f^{\sharp} d \hat{Z}_{j}\right)_{p}, X_{p}\right\rangle & =\left\langle\left(f^{\sharp} d \hat{Z}_{j}\right)_{p}, f_{*} X_{p}\right\rangle \\
& =\left\langle\left(d \hat{Z}_{j}\right)_{f(p)}, f_{*} X_{p}\right\rangle \\
& =\left\langle f^{*}\left(d \hat{Z}_{j}\right)_{p}, X_{p}\right\rangle \\
& =\left\langle d\left(\hat{Z}_{j} \circ f\right)_{p}, X_{p}\right\rangle,
\end{aligned}
$$

so that actually $\phi f^{\sharp} d \hat{Z}_{j}=d\left(\hat{Z}_{j} \circ f\right)$, which is CR by Lemma 10. The second claim follows from the same calculation. 
By an appropriate choice of basis $\theta^{a}, \theta$, and similarly on $\hat{M}$, we may assume that $R_{\bar{a} c}^{b}, R_{\bar{a}}^{b}, \hat{R}_{\bar{A} C}^{B}, R_{\bar{A}}^{B}$ are all 0 (cf. [5, Lemma 1.33). By Lemma 13, it follows that $S_{\bar{a} c}^{B}=S_{\bar{a}}^{B}=0$. Lemma 17 implies the following relations:

$$
\begin{aligned}
\xi h_{\bar{a} b} & =\gamma_{a}^{B} g_{\bar{b} B} ; \\
L_{\bar{a}} \xi+\xi h_{\bar{a}} & =g_{\bar{a} B} \eta^{B}+g_{\bar{a}} \xi .
\end{aligned}
$$

This follows comparing the coefficients of $D_{\bar{a}} \phi \tau$ and $\phi D_{\bar{a}} \tau$. We also need the following equalities:

$$
\begin{gathered}
L_{\bar{a}} \gamma_{b}^{B}=-\eta^{B} h_{\bar{a} b}, \\
L_{\bar{a}} \eta^{B}=-\eta^{B} h_{\bar{a}}, \\
T \gamma_{a}^{A}=L_{a} \eta^{A}+\eta^{A} h_{a},
\end{gathered}
$$

which follow as in $[5]$. We observe that the identity (18) implies that if $f$ is transversal at $p$ (i.e. $\xi(p) \neq 0$ ) and $M$ is Levi nondegenerate at $p$ (i.e. the matrix $\left(h_{\bar{a} b}\right)(p)$ is invertible), then the rank of the matrix $\left(\gamma_{a}^{B}\right)(p)$ must be maximal, i.e. $=n$. Thus, if $f$ is transversal and $M$ is Levi nondegenerate, then $f$ is in fact a local embedding.

\section{JET REFLECTION IDENTITIES FOR NONDEGENERATE MAPPINGS}

In this section we are going to establish the jet reflection identities necessary to use the machinery developed in [5]. Our first goal is the reflection identities for nondegenerate mappings. Fix an integer $k_{0}$. We denote by $\mathcal{K}$ the set of all strings $\mathbb{A}=\bar{a}_{1} \ldots \bar{a}_{k}$ of elements of $\{\overline{1}, \ldots, \bar{n}\}$ with $k \leq k_{0}$, and we write $\mathcal{K}_{\hat{n}}$ for the set of all $\hat{n}$-tuples of elements of $\mathcal{K}$. For $\mathbb{A}=\bar{a}_{1} \ldots \bar{a}_{k}$ we let $\ell(\mathbb{A})=k$ denote the length of $\mathbb{A}$.

Theorem 18. Let $k_{0} \in \mathbb{N}$. There exist functions

$$
\Delta_{\mathbf{\Lambda}}\left(\overline{L^{J} \gamma_{c}^{D}}\right), \quad r_{a, \mathbf{\Lambda}}^{B}\left(\overline{L^{J} \gamma_{c}^{D}}, \overline{L^{I} \xi}\right), \quad r_{\mathbf{\Delta}}^{B}\left(\overline{L^{J} \gamma_{c}^{D}}, \overline{L^{I} \xi}\right),
$$

where $|J| \leq k_{0}-1,|I| \leq k_{0}$, for $\mathbf{\Delta}=\left(\mathbf{A}_{0}, \ldots, \mathbf{A}_{n}\right) \in \mathcal{K}_{\hat{n}}^{n+1}, a \in\{1, \ldots, n\}$ and $B \in\{1, \ldots, \hat{n}\}$ with the following property: If $f: M \rightarrow \hat{M}$ is $k$-nondegenerate at 0 with $k \leq k_{0}$, then there exists $\mathbf{\Delta}^{0}$ with $\Delta_{\mathbf{\Delta}^{0}}\left(\overline{L^{J} \gamma_{c}^{D}}(0)\right) \neq 0$, and for any $\boldsymbol{\Lambda} \in \mathcal{K}_{\hat{n}}^{n+1}$ with $\Delta_{\mathbf{\Lambda}}\left(\overline{L^{J} \gamma_{c}^{D}}(0)\right) \neq 0$ the following equalities hold:

$$
\begin{gathered}
\gamma_{a}^{B}=r_{a, \mathbf{\Lambda}}^{B}\left(\overline{L^{J} \gamma_{c}^{D}}, \overline{L^{I} \xi}\right), \\
\eta^{B}=r_{\boldsymbol{\Delta}}^{B}\left(\overline{L^{J} \gamma_{c}^{D}}, \overline{L^{I} \xi}\right) .
\end{gathered}
$$

The $\Delta_{\mathbf{\Lambda}}$ are polynomials in $\overline{L^{J} \gamma_{c}^{D}}$ with complex coefficients; the functions $r_{a, \mathbf{\Lambda}}^{B}$ and $r_{\mathbf{\Lambda}}^{B}$ are rational in $\overline{L^{J} \gamma_{c}^{D}}$, polynomial in $\overline{L^{I} \xi}$, with coefficients smooth on $M \times \hat{M}$. Furthermore, $\Delta_{\mathbf{\Lambda}}, r_{a, \mathbf{\Lambda}}^{B}$, and $r_{\mathbf{\Lambda}}^{B}$ only depend on $M, \hat{M}$, and $k_{0}$ (and not on the mapping $f$ ).

In order to define the functions $\Delta_{\boldsymbol{\Lambda}}$ of the Theorem, we need the following computational Lemma.

Lemma 19. Let $f: M \rightarrow \hat{M}$ be a CR mapping. Then for each $k \in \mathbb{N}$, all $a \in\{1, \ldots, n\}$, and all $\mathbb{A}=\bar{a}_{1} \ldots \bar{a}_{k}$ the following identities hold:

$$
g_{\mathbb{A} B} \eta^{B}=\sum_{\ell\left(\mathbb{A}^{\prime}\right)<\ell(\mathbb{A})} g_{\mathbb{A}^{\prime} B} \eta^{B} v_{\mathbb{A}}^{\mathbb{A}^{\prime}}\left(\overline{L^{J} \gamma_{c}^{D}}\right)+w_{\mathbb{A}}\left(\overline{L^{J} \gamma_{c}^{D}}, \overline{L^{I} \xi}\right) ;
$$




$$
\begin{aligned}
g_{\mathbb{A} B} \gamma_{a}^{B}= & \sum_{\ell\left(\mathbb{A}^{\prime}\right)<\ell(\mathbb{A})} g_{\mathbb{A}^{\prime} B} \gamma_{a}^{B} p_{a \mathbb{A}}^{\mathbb{A}^{\prime}}\left(\overline{L^{J} \gamma_{c}^{D}}\right)+ \\
& \sum_{\ell\left(\mathbb{A}^{\prime}\right)<\ell(\mathbb{A})} g_{\mathbb{A}^{\prime}} \eta^{B} q_{a \mathbb{A}}^{\mathbb{A}^{\prime}}\left(\overline{L^{J} \gamma_{c}^{D}}\right)+r_{a \mathbb{A}}\left(\overline{L^{J} \gamma_{c}^{D}}, \overline{L^{I} \xi}\right) ;
\end{aligned}
$$

where $|J| \leq k-1,|I| \leq k$. The functions $v, w, p, q$, and $r$ are rational in $\overline{L^{I} \gamma_{c}^{D}}$, the functions $w$ and $r$ are polynomial in $\overline{L^{I} \xi}$, with coefficients smooth on $M \times \hat{M}$. Furthermore, $v, w, p, q$, and $r$ only depend on $M$ and $\hat{M}$.

Proof. We start with (25). For $k=1$, by (19),

$$
g_{\bar{a} B} \eta^{B}=L_{\bar{a}} \xi+\xi h_{\bar{a}}-\xi g_{\bar{a}}
$$

and recalling (11), $g_{\bar{a}}=\overline{\gamma_{a}^{A}} h_{\mathbb{A}}$, we have that

$$
g_{\bar{a} B} \eta^{B}=L_{\bar{a}} \xi+\xi h_{\bar{a}}-\xi \overline{\gamma_{a}^{A}} h_{\mathbb{A}}=w_{\bar{a}}\left(\overline{\gamma_{c}^{D}}, \overline{L^{I} \xi}\right),
$$

which is (25) for $k=1$. To get (25) for $k>1$, we use (25) for $k-1$ to express $g_{\bar{a}_{1} \ldots \bar{a}_{k-1} B} \eta^{B}$ and apply $L_{\bar{a}_{k}}$, using Lemma 14 and (21).

The proof of (26) is similar: We start with (18) for the case $k=1$, and for $k>1$, we use induction, Lemma 14, (20), and (21).

Proof of Theorem 18. Let $\mathbf{A}=\left(\mathbb{A}_{1}, \ldots, \mathbb{A}_{\hat{n}}\right)$. We rewrite the $\hat{n}$ equations obtained from (25) by replacing $\mathbb{A}$ with $\mathbb{A}_{1}, \ldots, \mathbb{A}_{\hat{n}}$ in the following form:

$$
M_{\mathbf{A}} \eta=w_{\mathbf{A}}
$$

where

$$
\eta=\left(\begin{array}{c}
\eta^{1} \\
\vdots \\
\eta^{\hat{n}}
\end{array}\right), \quad w=\left(\begin{array}{c}
w_{\mathbb{A}_{1}} \\
\vdots \\
w_{\mathbb{A}_{\hat{n}}}
\end{array}\right),
$$

and the $(i, B)$ th entry of $M_{\mathbf{A}}$ is

$$
g_{\mathbb{A}_{i} B}-\sum_{\ell\left(\mathbb{A}^{\prime}\right)<\ell\left(\mathbb{A}_{i}\right)} g_{\mathbb{A}^{\prime} B} v_{\mathbb{A}_{i}}^{\mathbb{A}^{\prime}}
$$

Similarly, for each $a \in\{1, \ldots, n\}$ we rewrite (26) as

$$
N_{a, \mathbf{A}} \gamma_{a}=K_{a, \mathbf{A}} \eta+r_{\mathbf{A}}
$$

By Lemma 16 and Lemma 19 the matrices $M_{\mathbf{A}}$ and $N_{a, \mathbf{A}}$ are polynomial in $\overline{L^{J} \gamma_{c}^{D}}$, with coefficients smooth on $M \times \hat{M}$ and they only depend on $M$ and $\hat{M}$. We define $\Delta_{0, \mathbf{A}}=\operatorname{det} M_{\mathbf{A}}$ and $\Delta_{j, \mathbf{A}}=\operatorname{det} N_{j, \mathbf{A}}$, $j=1, \ldots, n$, where we evaluate the coefficients of $\overline{L^{J} \gamma_{c}^{D}}$ at $(0,0)$, and for $\boldsymbol{\Delta}=\left(\mathbf{A}_{0}, \ldots, \mathbf{A}_{n}\right)$, we set $\Delta_{\mathbf{\Lambda}}=\prod_{j=0}^{n} \Delta_{j, \mathbf{A}_{j}}$. By Cramer's rule, if $\Delta_{\mathbf{\Lambda}}\left(\overline{L^{J} \gamma_{c}^{D}}(0)\right) \neq 0$, in a neighbourhood of 0 we can solve the equations (27) with $\mathbf{A}=\mathbf{A}_{0}$ and (28) with $(a, \mathbf{A})=\left(a_{1}, \mathbf{A}_{1}\right), \ldots,\left(a_{n}, \mathbf{A}_{n}\right)$ for $\eta^{B}$ and $\gamma_{a}^{B}$ in the required form.

We finish the proof by showing that if $f$ is $k$-nondegenerate at 0 with $k \leq k_{0}$, then there exists $\mathbf{\Lambda}^{0}$ with $\Delta_{\mathbf{\Delta}^{0}}\left(\overline{L^{J} \gamma_{c}^{D}}(0)\right) \neq 0$. First recall that by Lemma 15, $f$ is $k$-nondegenerate at 0 if and only if

$$
\operatorname{span}\left\{\left(g_{\mathbb{A} B}(0)\right)_{B}: \ell(\mathbb{A}) \leq k\right\}=\mathbb{C}^{\hat{n}} .
$$


Consider a system of vectors of the form

$$
v_{\mathbb{A}}=\left(g_{\mathbb{A} B}(0)-\sum_{\ell\left(\mathbb{A}^{\prime}\right)<\ell(\mathbb{A})} c_{\mathbb{A} B}^{\mathbb{A}^{\prime}} g_{\mathbb{A}^{\prime} B}(0)\right)_{B}, \quad \ell(\mathbb{A}) \leq k .
$$

We claim that if $f$ is $k$-nondegenerate, then $\operatorname{span}\left\{v_{\mathbb{A}}: \ell(\mathbb{A}) \leq k\right\}=\mathbb{C}^{\hat{n}}$, which implies that $\Delta_{\mathbf{\Delta}^{0}}\left(\overline{L^{J} \gamma_{c}^{D}}(0)\right) \neq$ 0 for some $\mathbf{\Lambda}^{0}$. To prove the last claim, we let $V_{l}=\operatorname{span}\left\{\left(g_{\mathbb{A} B}\right)_{B}(0): \ell(\mathbb{A}) \leq l\right\}$ and $W_{l}=\operatorname{span}\left\{v_{\mathbb{A}}: \ell(\mathbb{A}) \leq\right.$ $l\}$. By (30), $V_{1}=W_{1}$. But then, again by (30), $V_{2}=W_{2}$. By induction, we see that $W_{k}=V_{k}=\mathbb{C}^{\hat{n}}$.

Theorem 18 is completely analogous to Theorem 2.4 in $[5]$. The proof of Theorem 7 can now easily be completed using the arguments in [5] and a result from [8].

Proof of Theorem . By repeating the arguments in [5], based on the identities in Theorem 18 instead of those in Theorem 2.4 in [5], we obtain a complete system of some order $l_{0}$ (the exact value of which is not relevant for this argument) for $f$ and $g$. That is, there exists a neighbourhood $U$ of $p_{0}$ and a smooth function $\phi: U \times J^{l_{0}}(U, \hat{M}) \rightarrow J^{l_{0}+1}(U, \hat{M})$ such that

$$
j_{x}^{l_{0}+1} f=\phi\left(x, j_{x}^{l_{0}} f\right) \text { and } j_{x}^{l_{0}+1} g=\phi\left(x, j_{x}^{l_{0}} g\right), \quad x \in U .
$$

By Theorem 2 in [8], $j_{p_{0}}^{l} f=j_{p_{0}}^{l} g$ for all $l$ and, in particular then, $j_{p_{0}}^{l_{0}} f=j_{p_{0}}^{l_{0} g}$. The conclusion of Theorem 7 follows from the uniqueness of solutions of complete systems (see e.g. 四).

\section{JET REFLECTION IDENTITIES FOR LEVI-NONDEGENERATE HYPERSURFACES}

In this section, we consider the case $\hat{N}=N+1$, where $M$ is Levi-nondegenerate at 0 , and $\hat{M}$ is Levi-nondegenerate at $\hat{0}$. We are also assuming that $f$ is transversal, which in our notation is equivalent to $\xi(0) \neq 0$.

For the proof of Theorem 1 we first note that by Lemma 20 in $[8]$, the degeneracy of $f$ is either 0 or 1 . In the case where the degeneracy of $f$ is 0 , Theorem 1 follows from Theorem 0 , so that we only have to deal with the case where $f$ is constantly 1-degenerate. Using (18) it is easy to see that with our assumptions, the vectors $g_{a B}(0), a=1, \ldots, n$ are linearly independent, as are the vectors $\gamma_{c}^{D}(0)$, $c=1, \ldots, n$. We conclude that $f$ is constantly $(1,1)$-degenerate. Our goal in this section is to derive the following jet reflection identity for such mappings. The unique determination theorem will be proved in section 7 by producing a complete system using this jet reflection identity.

Theorem 20. Let $M \subset \mathbb{C}^{N}$ and $\hat{M} \subset \mathbb{C}^{N+1}$ be hypersurfaces which are Levi-nondegenerate at the points $p_{0}$ and $\hat{p}_{0}, f: M \rightarrow \hat{M}$ a smooth $C R$ map which is constantly $(1,1)$-degenerate and transversal at $p_{0}$. Then for any $I$ with $|I|=k$ there exist functions $r_{b}^{I, A}$ and $s^{I, A}$ for $a, b \in\{1, \ldots, n\}$ and $A \in\{1, \ldots, n+1\}$ such that

$$
\begin{aligned}
& L^{I} \gamma_{b}^{A}=r_{b}^{I, A}\left(L^{M} \gamma_{c}^{D}, L^{M} \eta^{D}, \overline{\gamma_{c}^{D}}, \overline{\eta^{D}}\right), \\
& L^{I} \eta^{A}=s^{I, A}\left(L^{M} \gamma_{c}^{D}, L^{M} \eta^{D}, \overline{L_{d} \gamma_{c}^{D}}, \overline{L_{d} \eta^{D}}, \overline{T \gamma_{c}^{D}}, \overline{T \eta^{D}}\right)
\end{aligned}
$$

Here $|M| \leq k-1$. The $r_{b}^{I, A}$ and $s^{I, A}$ are rational in their arguments with coefficients which are smooth on $M \times \hat{M}$, and they only depend on $M$ and $\hat{M}$.

Proof. Since $\hat{M}$ is Levi-nondegenerate, we can choose the basis of CR vector fields tangent to $\hat{M}$ such that

$$
\hat{h}_{\bar{A} B}(0)=\epsilon_{\bar{A} B}=\left\{\begin{array}{ll} 
\pm i, & A=B \\
0, & A \neq B
\end{array} .\right.
$$


Since $f$ is constantly $(1,1)$-degenerate, for any $\bar{a}, \bar{b}$ the determinant of the matrix

$$
\left(\begin{array}{ccc}
g_{\overline{1} 1} & \ldots & g_{\overline{1} n+1} \\
\vdots & & \vdots \\
g_{\bar{n} 1} & \ldots & g_{\bar{n} n+1} \\
L_{\bar{a}} g_{\bar{b} 1} & \ldots & L_{\bar{a}} g_{\bar{b} n+1}
\end{array}\right)
$$

vanishes. Let us write $(-1)^{B+1} \Delta^{B}$ for the determinant of the matrix obtained from the matrix (34) by dropping the last row and the $B$ th column. We fix $a$ and $b$ for the moment. By developing the determinant of (34) along the last row,

$$
\Delta^{B} L_{\bar{a}} g_{\bar{b} B}=0
$$

We compute

$$
L_{\bar{a}} g_{\bar{b} B}=L_{\bar{a}}\left(\overline{\gamma_{b}^{A}} \hat{h}_{\bar{A} B}\right)=\overline{L_{a} \gamma_{b}^{A}} \hat{h}_{\bar{A} B}+\overline{\gamma_{b}^{A} \gamma_{a}^{D}} \hat{L}_{\bar{D}} \hat{h}_{\bar{A} B} .
$$

Plugging (36) into (35) and using Lemma 16 we conclude that

$$
\Delta^{B} \overline{L_{a} \gamma_{b}^{A}} \hat{h}_{\bar{A} B}=r\left(\overline{\gamma_{c}^{D}}\right)
$$

where $r$ is rational in $\overline{\gamma_{c}^{D}}$ with coefficients smooth on $M \times \hat{M}$ and $r$ is determined only by $M$ and $\hat{M}$. We take the complex conjugate of this equation to obtain

$$
\Delta^{\bar{B}} L_{a} \gamma_{b}^{A} \hat{h}_{A \bar{B}}=\bar{r}\left(\gamma_{c}^{D}\right)
$$

Consider now the equations (18) which we rewrite as

$$
\xi h_{\bar{d} b}=\gamma_{b}^{A} g_{\bar{d} A} .
$$

We apply $L_{a}$ to this equation and conclude, using also equations (18) and (19),

$$
\left(L_{a} \gamma_{b}^{A}\right) g_{\bar{d} A}=\tilde{p}_{\bar{d}}\left(\gamma_{c}^{D}, \overline{\gamma_{c}^{D}}, \xi, L_{a} \xi\right)=p_{\bar{d}}\left(\gamma_{c}^{D}, \eta^{D} \overline{\gamma_{c}^{D}}, \overline{\eta^{D}}\right),
$$

where $p_{\bar{d}}$ is rational in its arguments with coefficients smooth on $M \times \hat{M}$ and $p_{\bar{d}}$ is determined only by $M$ and $\hat{M}$. We claim that the matrix

$$
\left(\begin{array}{ccc}
g_{\overline{1} 1}(0) & \ldots & g_{\overline{1} n+1}(0) \\
\vdots & & \vdots \\
g_{\bar{n} 1}(0) & \ldots & g_{\bar{n} n+1}(0) \\
\Delta^{\overline{1}}(0) \epsilon_{\overline{1} 1} & \ldots & \Delta^{\overline{n+1}}(0) \epsilon_{\overline{n+1} n+1}
\end{array}\right)
$$

is invertible, so that the system of equations

$$
\begin{aligned}
X^{A} g_{\bar{d} A} & =p_{\bar{d}}, \quad d \in\{1, \ldots, n\}, \\
X^{A} \Delta^{\bar{B}} \hat{h}_{A \bar{B}} & =\bar{r},
\end{aligned}
$$

can be solved uniquely for the $X^{A}$ (in a neighbourhood of 0 ). Developing the determinant $\Delta$ for the matrix (40) along the last row, we conclude that

$$
\Delta=\epsilon_{\bar{A} A} \Delta^{\bar{A}} \Delta^{A} .
$$


We are now going to show that this last expression is not equal to 0 . Consider the matrices

$$
G=\left(\begin{array}{ccc}
g_{\overline{1} 1}(0) & \ldots & g_{\overline{1} n+1}(0) \\
\vdots & & \vdots \\
g_{\bar{n} 1}(0) & \ldots & g_{\bar{n} n+1}(0)
\end{array}\right), \quad \Gamma=\left(\begin{array}{ccc}
\gamma_{\overline{1}}^{1}(0) & \ldots & \gamma_{\bar{n}}^{1}(0) \\
\vdots & & \vdots \\
\gamma_{\overline{1}}^{n+1}(0) & \ldots & \gamma_{\bar{n}}^{n+1}(0),
\end{array}\right)
$$

and the $n \times n$ matrix

$$
H=\left(\begin{array}{ccc}
h_{\overline{1} 1}(0) & \ldots & h_{\overline{1} n}(0) \\
\vdots & & \vdots \\
h_{\bar{n} 1}(0) & \ldots & h_{\bar{n} n}(0)
\end{array}\right) .
$$

In matrix notation, (18) then reads as $\xi H=G \Gamma$. Let us write $G_{A}$ for the matrix $G$ with the $A$ th column dropped, and $\Gamma^{A}$ for the matrix $\Gamma$ with the $A$ th row dropped. The Cauchy-Binet Theorem from linear algebra states that $\operatorname{det} G \Gamma^{t}=\operatorname{det} G_{A} \operatorname{det} \Gamma^{A}$. By our convention (33) and (11) it follows that

$$
g_{\bar{a} B}(0)=\overline{\gamma_{a}^{B}}(0) \epsilon_{\bar{B} B} .
$$

Writing $\epsilon=\prod_{B} \epsilon_{\bar{B} B}$ we obtain from the equation above

$$
\Delta^{A}=\frac{\epsilon}{\epsilon_{\bar{A} A}} \operatorname{det} \overline{\Gamma^{A}} .
$$

Recalling that $\Delta^{A}=\operatorname{det} G_{A}$, we see that the Cauchy-Binet Theorem implies that

$$
\begin{aligned}
\Delta & =\epsilon_{\bar{A} A} \Delta^{\bar{A}} \Delta^{A} \\
& =\epsilon \operatorname{det} \overline{G_{A}} \operatorname{det} \overline{\Gamma^{A}} \\
& =\epsilon \xi(0)^{n} \operatorname{det} H \neq 0,
\end{aligned}
$$

and our last claim is proved. Using that the matrix (40) is invertible to solve (38) and (39) for $L_{a} \gamma_{b}^{A}$, we obtain (by repeatedly differentiating the resulting equations and using the identities (20), (21), and (22)) the first part of Theorem 20.

We also need reflection identities (32) for $L_{a} \eta^{B}$. We will deduce those from the equation (31) we just proved. We first compute

$$
L_{\bar{c}} L_{b} \gamma_{e}^{B}=L_{b} L_{\bar{c}} \gamma_{e}^{B}-h_{\bar{c} b} T \gamma_{e}^{B},
$$

which follows from (9) and the fact that $R_{\bar{a} c}^{b}=0$ (cf. [5], Lemma 1.33). By (20) and (22), we conclude that

$$
L_{\bar{c}} L_{b} \gamma_{e}^{B}=-L_{b}\left(\eta^{B} h_{\bar{c} e}\right)-h_{\bar{c} b}\left(L_{e} \eta^{B}+\eta^{B} h_{e}\right)=-\left(h_{\bar{c} e} L_{b} \eta^{B}+h_{\bar{c} b} L_{e} \eta^{B}\right)+r\left(\eta^{B}\right),
$$

where $r\left(\eta^{B}\right)$ is a (linear) polynomial in $\eta^{B}$ with coefficients which are smooth on $M$ (and only depend on $M$ ). The identity (32) with $k=1$ now follows by solving for $L_{b} \eta^{B}$ in (46) (e.g. set $b=c=e$ and recall that $h_{\bar{b} b} \neq 0$ ) and then applying $L_{\bar{c}}$ to (31) with $k=1$, and finally using (20) and (21) to substitute for $L_{\bar{c}} \gamma_{e}^{B}$ and $L_{\bar{c}} \eta^{B}$. The general case follows by differentiating. The details are left to the reader.

\section{Strictly PSEUdoconVEX TARGETS}

Our goal in this section is to derive the following reflection identity; in section 7 we will use it to derive a complete system for the mapping $f$. 
Theorem 21. Let $M \subset \mathbb{C}^{N}, \hat{M} \subset \mathbb{C}^{\hat{N}}$ be smooth hypersurfaces, $p_{0} \in M, \hat{p}_{0} \in \hat{M}$, with $\hat{M}$ strictly pseudoconvex at $\hat{p}_{0}$. Also assume that $f$ is a smooth $C R$ mapping from $M$ to $\hat{M}$ with $f\left(p_{0}\right)=\hat{p}_{0}$ which is transversal and constantly $\left(k_{0}, s\right)$-degenerate at $p_{0}$. Then for any $I$ with $|I|=k, a \in\{1, \ldots, n\}$, $B \in\{1, \ldots, \hat{n}\}$ there exist functions $r_{a}^{I, B}$ and $s^{I, B}$ which are rational in their arguments such that

$$
\begin{aligned}
& L^{I} \gamma_{a}^{B}=r_{a}^{I, B}\left(L^{M} T^{l} \gamma_{c}^{D}, L^{M} T^{l} \eta^{D}, \overline{L^{J} T^{m} \gamma_{c}^{D}}, \overline{L^{J} T^{m} \eta^{D}}\right), \\
& L^{I} \eta^{B}=s^{I, B}\left(L^{M} T^{l} \gamma_{c}^{D}, L^{M} T^{l} \eta^{D}, \overline{L^{J} T^{m} \gamma_{c}^{D}}, \overline{L^{J} T^{m} \eta^{D}}\right) .
\end{aligned}
$$

Here $l \in\{0,1\},|M|+l \leq \max \left(k_{0}, k-1\right),|J|+m \leq k_{0}+1, m \leq \min \left(k, k_{0}\right)$. Furthermore, $r_{a}^{I, B}$ and $s^{I, B}$ only depend on $M, \hat{M}$, and $j_{p_{0}}^{k_{0}} f$.

Proof. Assuming that $\hat{M}$ is strictly pseudoconvex, we choose a local basis of CR vector fields tangent to $\hat{M}$ such that $\hat{h}_{\bar{A} B}(0)=i \delta_{A}^{B}$. Since $f$ is transversal, it follows that $M$ is strictly pseudoconvex at 0 , and we likewise choose a local basis of CR vector tangent to $M$ such that $h_{\bar{a} b}(0)=i \delta_{a}^{b}$. As in Theorem 2, we assume that $f$ is constantly $\left(k_{0}, s\right)$-degenerate at $p_{0}$. We let

$$
V_{k}=\operatorname{span}\left\{g_{\mathbb{A} B}(0): \ell(\mathbb{A}) \leq k\right\}
$$

and claim that we can assume that $V_{k_{0}} \subset \mathbb{C}^{\hat{n}}$ is just the subspace spanned by the first $s$ unit vectors. To see this, recall that

$$
g_{\bar{a}_{1} \ldots \bar{a}_{k} B}=\left\langle D_{\bar{a}_{k}} D_{\bar{a}_{k-1}} \ldots D_{\bar{a}_{1}} \tau, \hat{L}_{B}\right\rangle .
$$

Hence, under a change of basis $\hat{L}_{B}^{\prime}=u_{B}^{C} \hat{L}_{C}$, the $g$ transform as $g_{\mathbb{A} B}^{\prime}=u_{B}^{D} g_{\mathbb{A} D}$. Also, since

$$
\hat{h}_{\bar{A} B}=\left\langle d \hat{\theta}, \hat{L}_{\bar{A}} \wedge \hat{L}_{B}\right\rangle,
$$

the $\hat{h}_{\bar{A} B}$ transform as $\hat{h}_{\bar{A} B}^{\prime}=\bar{u}_{A}^{C} u_{B}^{D} \hat{h}_{\bar{C} D}$, and any $u_{A}^{B}$ which is unitary at 0 will respect the normalization condition $\hat{h}_{\bar{A} B}(0)=i \delta_{A}^{B}$. So, our claim is proved.

Since $f$ is constantly $\left(k_{0}, s\right)$-degenerate, we can choose $\mathbb{A}_{1, a}, \ldots, \mathbb{A}_{s, a}$ for $a \in\{1, \ldots, n\}$ and $\mathbb{A}_{1}, \ldots, \mathbb{A}_{s}$, whose length does not exceed $k_{0}$, with such that for any $a \in\{1, \ldots, n\}$ the vectors

$$
v_{a, j}=\left(g_{\mathbb{A}_{j, a} B}-\sum_{\ell\left(\mathbb{A}^{\prime}\right)<\ell\left(\mathbb{A}_{j, a}\right)} g_{\mathbb{A}^{\prime} B} v_{\mathbb{A}_{j}^{\mathbb{A}^{\prime}}}^{\mathbb{A}^{\prime}}\left(\overline{L^{J} \gamma_{c}^{D}}\right)\right), \quad j=1, \ldots, s,
$$

as well as

$$
v_{j}=\left(g_{\mathbb{A}_{j} B}-\sum_{\ell\left(\mathbb{A}^{\prime}\right)<\ell\left(\mathbb{A}_{j}\right)} g_{\mathbb{A}^{\prime} B} p_{a \mathbb{A}_{j}}^{\mathbb{A}^{\prime}}\left(\overline{L^{J} \gamma_{c}^{D}}\right)\right), \quad j=1, \ldots, s,
$$

are linearly independent at 0 , and hence each of these $n+1$ families of vectors span $V_{k_{0}}$. For any $t \in\{1, \ldots, n\}, C \in\{1, \ldots, \hat{n}\}$, and $I=\left(I_{1}, \ldots, I_{k}\right)$, the determinants of

$$
\left(\begin{array}{cccc}
v_{a, 1}^{1} & \ldots & v_{a, 1}^{s} & v_{a, 1}^{C} \\
\vdots & & \vdots & \vdots \\
v_{a, s}^{1} & \ldots & v_{a, s}^{s} & v_{a, s}^{C} \\
L^{\bar{I}} g_{\bar{a} 1} & \ldots & L^{\bar{I}} g_{\bar{a} s} & L^{\bar{I}} g_{\bar{a} C}
\end{array}\right), \quad a=1, \ldots, n
$$


and

$$
\left(\begin{array}{cccc}
v_{1}^{1} & \ldots & v_{1}^{s} & v_{1}^{C} \\
\vdots & & \vdots & \vdots \\
v_{s}^{1} & \ldots & v_{s}^{s} & v_{s}^{C} \\
L^{\bar{I}} g_{\bar{t} 1} & \ldots & L^{\bar{I}} g_{\bar{t} s} & L^{\bar{I}} g_{\bar{t} C}
\end{array}\right)
$$

vanish on $M$. Since

$$
L^{\bar{I}} g_{\bar{t} B}=\overline{L^{I}\left(\gamma_{t}^{A} \hat{h}_{A \bar{B}}\right)}=\overline{L^{I} \gamma_{t}^{A}} \hat{h}_{\bar{A} B}+q_{t, B}^{I}\left(\overline{L^{J} \gamma_{c}^{D}}\right),
$$

where $|J|<k$, expanding (49) and (50) along the last row we obtain

$$
\begin{gathered}
\Delta_{\bar{a}, C_{0}}^{\bar{b}}\left(L^{I} \gamma_{a}^{A}\right) \hat{h}_{A \bar{b}}+\bar{\Delta}_{a}\left(L^{I} \gamma_{a}^{A}\right) \hat{h}_{A \bar{C}_{0}}=r_{a, C_{0}}^{I}\left(L^{J} \gamma_{a}^{B}\right), \quad a=1, \ldots, n, \\
\Delta_{\bar{C}_{0}}^{\bar{b}}\left(L^{I} \gamma_{t}^{A}\right) \hat{h}_{A \bar{b}}+\bar{\Delta}\left(L^{I} \gamma_{t}^{A}\right) \hat{h}_{A \bar{C}_{0}}=r_{C_{0}}^{I}\left(L^{J} \gamma_{a}^{B}\right)
\end{gathered}
$$

where again $|J|<k, r_{a, C_{0}}^{I}$ and $r_{C_{0}}^{I}$ are polynomial in their arguments with smooth coefficients, and $\Delta_{\bar{a}, C_{0}}^{\bar{b}}$, $\bar{\Delta}_{a}, \Delta_{\bar{C}_{0}}^{\bar{b}}, \bar{\Delta}_{a}$ denotes the various cofactors produced by the row expansion. These are rational in $L^{J} \gamma_{a}^{B}$, where $|J| \leq k_{0}-1$, and polynomial in $L^{K} \xi$, where $|K| \leq k_{0}$.

We need the following computational fact, which the reader can verify easily.

Lemma 22. Given $P=p_{1} \ldots p_{k}$ with $1 \leq p_{j} \leq n$, the following holds for any $a$.

$$
L_{\bar{a}} L^{P}=L^{P} L_{\bar{a}}-\sum_{j=1}^{k} h_{\bar{a} p_{j}} L^{P_{j}} T+\sum_{|K| \leq k-2} C_{K} L^{K} T,
$$

where $P_{j}$ is obtained from $P$ by removing the $j$ th entry.

Now given any $I=i_{1} \ldots i_{k}$ with $i_{1} \leq i_{2} \leq \cdots \leq i_{k}$, we first apply the operator $L^{I}$ to the equations (25) and (26) to obtain

$$
\begin{aligned}
g_{\mathbb{A} B}\left(L^{I} \eta^{B}\right)-\sum_{\ell\left(\mathbb{A}^{\prime}\right)<\ell(\mathbb{A})} g_{\mathbb{A}^{\prime} B}\left(L^{I} \eta^{B}\right) v_{\mathbb{A}}^{\mathbb{A}^{\prime}}\left(\overline{L^{J} \gamma_{c}^{D}}\right) & \\
& =\tilde{w}_{\mathbb{A}}\left(L^{M} \gamma_{c}^{D}, \overline{L^{J} T^{m} \gamma_{c}^{D}}, \overline{L^{J} T^{m} \eta^{D}}\right)
\end{aligned}
$$

and

$$
\begin{aligned}
g_{\mathbb{A} B}\left(L^{I} \gamma_{a}^{B}\right)-\sum_{\ell\left(\mathbb{A}^{\prime}\right)<\ell(\mathbb{A})} & g_{\mathbb{A}^{\prime} B}\left(L^{I} \gamma_{a}^{B}\right) p_{a \mathbb{A}}^{\mathbb{A}^{\prime}}\left(\overline{L^{J} \gamma_{c}^{D}}\right)- \\
& \sum_{\ell\left(\mathbb{A}^{\prime}\right)<\ell(\mathbb{A})} g_{\mathbb{A}^{\prime} B}\left(L^{I} \eta^{B}\right) q_{a \mathbb{A}}^{\mathbb{A}^{\prime}}\left(\overline{L^{J} \gamma_{c}^{D}}\right)=\tilde{r}_{a \mathbb{A}}\left(L^{M} \gamma_{c}^{D}, \overline{L^{J} T^{m} \gamma_{c}^{D}}, \overline{L^{J} T^{m} \eta^{D}}\right),
\end{aligned}
$$

where $|M|<k,|K| \leq k_{0}-1,|J| \leq k_{0}, m \leq k$, and $|J|+m \leq k_{0}$. We have also simplified matters a bit by applying (18) in order to get rid of the terms involving $\xi$.

Let us recall (52):

$$
\Delta_{\bar{C}_{0}}^{\bar{b}}\left(L^{I} \gamma_{t}^{A}\right) \hat{h}_{A \bar{b}}+\bar{\Delta}\left(L^{I} \gamma_{t}^{A}\right) \hat{h}_{A \bar{C}_{0}}=r_{C_{0}}^{I}\left(L^{M} \gamma_{a}^{B}\right)
$$


where $|M| \leq k-1$. Applying $L_{\bar{t}}$ to this equation, and applying Lemma 22 and (22), we obtain

$$
\begin{gathered}
\left(L^{I} \gamma_{t}^{A}\right) L_{\bar{t}}\left(\Delta_{\bar{C}_{0}}^{\bar{b}} \hat{h}_{A \bar{b}}\right)+\left(L^{I} \gamma_{t}^{A}\right) L_{\bar{t}}\left(\bar{\Delta} \hat{h}_{A \bar{C}_{0}}\right)-\Delta_{\bar{C}_{0}}^{\bar{b}}\left(L^{I} \eta^{A}\right) h_{\bar{t} t} \hat{h}_{A \bar{b}} \\
-\bar{\Delta}\left(L^{I} \eta^{A}\right) h_{\bar{t} t} \hat{h}_{A \bar{C}_{0}}-\sum_{j=1}^{k} h_{\bar{t} i_{j}}\left(L^{I_{j}} L_{t} \eta^{A}\right) \Delta_{\bar{C}_{0}}^{\bar{b}} \hat{h}_{A \bar{b}}-\sum_{j=1}^{k} h_{\bar{t} i_{j}}\left(L^{I_{j}} L_{t} \eta^{A}\right) \bar{\Delta} \hat{h}_{A \bar{C}_{0}} \\
=\tilde{r}_{C_{0}}^{I}\left(L^{M} \eta^{B}, L^{M} \gamma_{a}^{B}, L^{J} T \eta^{B}, L^{J} T \gamma_{a}^{B}\right) .
\end{gathered}
$$

In this equation, $|M|<k$, and $|J|<k-1$.

We consider the system of linear equations in the $L^{I} \gamma_{a}^{B}$ and $L^{I} \eta^{B}$ where $I$ ranges over all $I=i_{1} \ldots i_{k}$ with $i_{1} \leq i_{2} \leq \cdots \leq i_{k}, a \in\{1, \ldots, n\}, B \in\{1 \ldots, \hat{n}\}$ which is obtained in the following way. In (54) we set $\mathbb{A}=\mathbb{A}_{1}, \ldots, \mathbb{A}_{s}$. For each $I$, this gives $s$ linear equations in $L^{I} \eta^{B}$. We next consider the $\hat{n}-s$ equations obtained from (56) by replacing $t$ by $i_{k}$ and letting $C_{0}=s+1, s+2, \ldots, \hat{n}$. For the equations in $L^{I} \gamma_{a}^{B}\left(a \in\{1, \ldots, n\}\right.$ fixed) we replace $\mathbb{A}$ in $(55)$ by $\mathbb{A}_{a, 1} \ldots \mathbb{A}_{a, s}$, which gives us (for each $I$ and each a) $s$ linear equations in $L^{I} \gamma_{a}^{B}$. For each $I$ and each $a$ we also consider the equations obtained from (52) by replacing $t$ by $a$, and letting $C_{0}=s+1, \ldots, \hat{n}$.

With the simplifications above, it is easy to see that this linear system can be solved for $L^{I} \gamma_{a}^{B}$ and $L^{I} \eta^{B}$. We can now lift the restriction that $I$ is increasing by noting that rearranging the order of the $i_{j} \mathrm{~s}$ only produces error terms of order less than $k$. This finishes the proof.

\section{Constructing the Complete system; Proof of Theorem 8}

We are now going to construct the complete system for the jets of $f$. Since Theorems 20 and 21 are a bit different than the reflection identities in [5], we will go into some detail. For the rest of this section we assume that we are either in the situation of Theorem 20 or in the situation of Theorem 21. First, let us recall the following fact from [5] (the second part of Proposition 3.18):

For any multi-index $J$, integer $k \geq 1$, and index $c \in\{1, \ldots, n\}$ there exist smooth functions $b_{s}^{e_{1} \ldots e_{m}}$ such that

$$
\sum_{m=1}^{|J|+k} \sum_{s=0}^{k} b_{s}^{e_{1} \ldots e_{m}} \underbrace{\left.\left[\ldots\left[L_{e_{1}} \ldots L_{e_{m}}, L_{\bar{c}}\right], L_{\bar{c}}\right] \ldots, L_{\bar{c}}\right]}_{\text {length } s}=\left(h_{\bar{c} 1}\right)^{p} L^{J} T^{k} .
$$

Here, $p=k+|J|-|J|_{1}+1,|J|_{1}$ denotes the number of occurences of the index 1 in the multi-index $J$, and the length of the commutator $\left.\left[\ldots\left[X, Y_{1}\right], Y_{2}\right] \ldots, Y_{s}\right]$ is $s$. Since in any case $M$ is Levi-nondegenerate at 0 , we can use this identity in order to find a formula for $L^{J} T^{k} \gamma_{a}^{B}$ and $L^{J} T^{k} \eta^{B}$. We first apply $L_{\bar{c}}$ (at most $k$ times) to $\gamma_{a}^{B}$ and $\eta_{a}^{B}$, and using equations (20) and (21) we see that the result is polynomial in $\gamma_{c}^{D}, \eta_{c}^{D}$, and $\overline{L_{\bar{c}}^{j} \gamma_{c}^{D}}$, where $j \leq k-1$. Next, we apply $L_{e_{1}} \ldots L_{e_{m}}$ to the result, and we apply our reflection identities (either (31) and (32) or (47) and (48)) to see that the result is rational in

$$
L^{M} T^{l} \gamma_{c}^{D}, L^{M} T^{l} \eta^{D}, \overline{L^{K} T^{m} \gamma_{c}^{D}}, \overline{L^{K} T^{m} \eta^{D}}
$$

where $|M|+l \leq \max \left(k_{0},|J|+k-1\right),|K|+m \leq k_{0}+1$, and $m \leq|J|$. Another application of $L_{\bar{c}}$ (at most $k$ times) then gives us that

$$
\begin{aligned}
& L^{J} T^{k} \gamma_{a}^{B}=u\left(L^{M} T^{l} \gamma_{c}^{D}, L^{M} T^{l} \eta^{D}, \overline{L^{K} T^{m} \gamma_{c}^{D}}, \overline{L^{K} T^{m} \eta^{D}}\right), \\
& L^{J} T^{k} \eta^{B}=v\left(L^{M} T^{l} \gamma_{c}^{D}, L^{M} T^{l} \eta^{D}, \overline{L^{K} T^{m} \gamma_{c}^{D}}, \overline{L^{K} T^{m} \eta^{D}}\right),
\end{aligned}
$$

where $|M|+l \leq \max \left(k_{0},|J|+k-1\right),|K|+m \leq k+k_{0}+1$, and $m \leq \min \left(|J|+k, k_{0}\right)$. We are abusing notation by denoting by $u, v$ and $w$ functions which are rational in their arguments with coefficients smooth on $M \times \hat{M}$ and which only depend on $M$ and $\hat{M}$ (and are allowed to change). Now in these 
equations for $|J|+k=2 k_{0}+2$ we can substitute for the conjugated terms, yielding the same equations, but with the bounds $|M|+l \leq 2 k_{0}+1,|K|+m \leq 2 k_{0}+1$. Another use of equations (20) and (21) lets us conclude that for all $J, k, R$ with $|J|+k+|R|=2 k_{0}+2$,

$$
\begin{aligned}
& L^{J} T^{k} L^{\bar{R}} \gamma_{a}^{B}=u\left(L^{M} T^{l} \gamma_{c}^{D}, L^{M} T^{l} \eta^{D}, \overline{L^{K} T^{m} \gamma_{c}^{D}}, \overline{L^{K} T^{m} \eta^{D}}\right), \\
& L^{J} T^{k} L^{\bar{R}} \eta^{B}=v\left(L^{M} T^{l} \gamma_{c}^{D}, L^{M} T^{l} \eta^{D}, \overline{L^{K} T^{m} \gamma_{c}^{D}}, \overline{L^{K} T^{m} \eta^{D}}\right),
\end{aligned}
$$

with $|M|+l \leq 2 k_{0}+1,|K|+m \leq 2 k_{0}+1$. These two equations together with (18) let us also see that for all $J, k, R$ with $|J|+k+|R|=2 k_{0}+2$,

$$
L^{J} T^{k} L^{\bar{R}} \xi=w\left(L^{M} T^{l} \gamma_{c}^{D}, L^{M} T^{l} \eta^{D}, \overline{L^{K} T^{m} \gamma_{c}^{D}}, \overline{L^{K} T^{m} \eta^{D}}\right),
$$

again with $|M|+l \leq 2 k_{0}+1,|K|+m \leq 2 k_{0}+1$. Equations (60), (61), and (62), written in some local coordinate systems, constitute the complete system of differential equations described in Theorem 8. Also note that the same complete system works for mappings whose jets are close to the $2 k_{0}+2$ jet of $f$. This finishes the proof of Theorem 8 .

\section{REFERENCES}

[1] M. S. Baouendi, P. Ebenfelt, and L. P. Rothschild. Local geometric properties of real submanifolds in complex space. Bull. Amer. Math. Soc. (N.S.), 37(3):309-336 (electronic), 2000.

[2] M. S. Baouendi, P. Ebenfelt, and L. P. Rothschild. Real submanifolds in complex space and their mappings. Princeton University Press, Princeton, NJ, 1999.

[3] M. S. Baouendi, X. Huang, and L. P. Rothschild. Regularity of CR mappings between algebraic hypersurfaces. Invent. Math., 125(1):13-36, 1996.

[4] R. L. Bryant, S. S. Chern, R. B. Gardner, H. L. Goldschmidt, and P. A. Griffiths. Exterior Differential Systems, volume 18 of Mathematical Sciences Research Institute Publications. Springer-Verlag, New York, 1991.

[5] P. Ebenfelt. Finite jet determination of biholomorphic mappings at the boundary. To appear, Asian J. Math.

[6] F. Forstnerič. Extending proper holomorphic mappings of positive codimension. Invent. Math., 95(1):31-61, 1989.

[7] P. Griffiths and J. Harris. Principles of algebraic geometry. John Wiley \& Sons Inc., New York, 1994. Reprint of the 1978 original.

[8] B. Lamel. Holomorphic maps of real submanifolds in complex spaces of different dimensions. Pacific J. Math., 201(2):357-387, 2001.

[9] B. Lamel. A reflection principle for real-analytic submanifolds of complex spaces. J. Geom. Anal., 11(4):625-631, 2001.

[10] D. Zaitsev and S.-Y. Kim. The equivalence and the embedding problems for cr-structures of any codimension. Preprint, math.CV/0108093, 2001.

Department of Mathematics, University of California at San Diego, La Jolla, CA 92093

E-mail address: pebenfel@math.ucsd.edu

Department of Mathematics, University of Illinois, Urbana

E-mail address: lamelb@member.ams.org 